\title{
Quantum Stochastic Processes
}

\author{
By \\ Luigi ACCARDI**, Alberto FRIGERIO** and John T. LEWIS****
}

\begin{abstract}
A class of non-commutative stochastic processes is defined. These processes are defined up to equivalence by their multi-time correlation kernels. A reconstruction theorem, generalizing the Kolmogorov theorem for classical processes, is proved. Markov processes and their associated semigroups are studied, and some non-quasi free examples are constructed on the Clifford algebra, with the use of a perturbation theory of Markov processes. The connection with the Hepp-Lieb models is discussed.
\end{abstract}

\section{§0. Introduction}

We study a class of non-commutative stochastic processes which are determined up to equivalence by their multi-time correlations. They are analogues of classical processes in the sense of Doob [1], Meyer [2]; indeed, those processes are included as a special case.

We define a stochastic process over a $C^{*}$-algebra $\mathscr{B}$, indexed by a set $T$, to consist of a $C^{*}$-algebra $\mathscr{A}$, a family $\left\{j_{t}: t \in T\right\}$ of $*$-homomorphisms from $\mathscr{B}$ into $\mathscr{A}$ and a state $\omega$ on $\mathscr{A}$. This structure gives rise to a non-commutative stochastic process in the sense of Accardi [3], with local algebras defined by $\mathscr{A}_{I}=\vee\left\{j_{t}(b): t \in I, b \in \mathscr{B}\right\}$ for any subset $I$ of $T$; observables which are "localized at different times" are not assumed to commute. We show (Proposition 1.1) that such a process is determined up to equivalence by its family of correlation kernels $\omega\left(j_{t_{1}}\left(a_{1}\right) * \cdots j_{t_{n}}\left(a_{n}\right)^{*} j_{t_{n}}\left(b_{n}\right) \cdots j_{t_{1}}\left(b_{1}\right)\right)$; these are obtained by polarization from the expressions $\omega\left(j_{t_{1}}\left(b_{1}\right)^{*} \cdots j_{t_{n}}\left(b_{n}\right)^{*} j_{t_{n}}\left(b_{n}\right) \cdots j_{t_{1}}\left(b_{1}\right)\right)$, which are positive real numbers, and are the analogues of the finite-dimensional joint distributions of classical probability; they can, in principle, be determined by

Communicated by H. Araki, November 20, 1980.

* Istituto Matematico dell' Università, Milano, Italy.

** Dublin Institute for Advanced Studies, 10 Burlington Road, Dublin 4, Ireland. On leave of absence from Istituto di Scienze Fisiche dell' Università, Milano, Italy.

*** Dublin Institute for Advanced Studies, 10 Burlington Road, Dublin 4, Ireland. 
sequences of measurement operations $[4,5,6]$, when $t_{1} \leqq t_{2} \leqq \cdots \leqq t_{n}$ (if $T$ represents time); the correlation kernels for arbitrary times can be deduced from the time-ordered ones when the commutation relations are known.

We prove (Theorem 1.3) that a process can be reconstructed up to equivalence from a projective family of correlation kernels; this is a generalization of Kolmogorov's theorem [7] (related results are given in Theorems 1.7.1, 1.7.2 and Corollary 1.8.2). Markov processes and their associated semigroups are defined; we investigate the connections between them (Theorems 2.1 and 2.2.2), and we develop a perturbation theory of Markov processes, based on a quantum Feynman-Kac formula [8,9] (Theorems 2.3 and 2.4.4). We construct some examples on the Clifford algebra. First we construct quasi-free processes and we characterize the quasi-free Markov processes by means of an analogu of Doob's theorem [10] (Theorems 3.2.1 to 3.3.2), then we perturb them using the perturbation theory of Markov processes of Theorems 2.3 and 2.4.4. We show that these processes satisfy a Langevin equation (Theorems 3.4 .1 and 3.4.2) and we indicate (in §4) how they arise in the Hepp-Lieb models $[11,12]$.

\section{§1. Stochastic Processes and the Reconstruction Theorem}

1.1. Let $\mathscr{B}$ be a $C^{*}$-algebra with identity and let $T$ be a set; a stochastic process over $\mathscr{B}$ indexed by $T$ is a triple $\left(\mathscr{A},\left\{j_{t}: t \in T\right\}, \omega\right)$ where $\mathscr{A}$ is a $C^{*}$-algebra with identity and, for each $t$ in $T, j_{t}$ is a ${ }^{*}$-homomorphism of $\mathscr{B}$ into $\mathscr{A}$ with $j_{t}\left(1_{\mathscr{R}}\right)=1_{\mathscr{A}}, \mathscr{A}$ is generated by the image algebras $\left\{\mathscr{A}_{t}=j_{t}(\mathscr{B}): t \in T\right\}$ and $\omega$ is a state on $\mathscr{A}$. Let $(\mathscr{H}, \pi, \Omega)$ be the GNS triple associated with $(\mathscr{A}, \omega)$. Two stochastic processes $\left(\mathscr{A}^{(i)},\left\{j_{t}^{(i)}\right\}, \omega^{(i)}\right), i=1,2$, over the same $C^{*}$-algebra $\mathscr{B}$ and indexed by the same set $T$, are said to be equivalent if there is a unitary operator $U: \mathscr{H}^{(1)} \rightarrow \mathscr{H}^{(2)}$ such that $U \Omega^{(1)}=\Omega^{(2)}$ and $U \pi^{(1)} j_{t}^{(1)}(b)=\pi^{(2)} j_{t}^{(2)}(b) U$ for all $b$ in $\mathscr{B}$ and $t$ in $T$.

Proposition 1.1. Two stochastic processes $\left(\mathscr{A}^{(i)},\left\{j_{t}^{(i)}\right\}, \omega^{(i)}\right), i=1,2$, over $\mathscr{B}$ indexed by $T$ are equivalent if and only if

$$
\begin{aligned}
\omega^{(1)}\left(j_{t_{1}}^{(1)}\left(a_{1}\right) * \cdots\right. & \left.j_{t_{n}}^{(1)}\left(a_{n}\right) * j_{t_{n}}^{(1)}\left(b_{n}\right) \cdots j_{t_{1}}^{(1)}\left(b_{1}\right)\right) \\
& =\omega^{(2)}\left(j_{t_{1}}^{(2)}\left(a_{1}\right) * \cdots j_{t_{n}}^{(2)}\left(a_{n}\right) * j_{t_{n}}^{(2)}\left(b_{n}\right) \cdots j_{t_{1}}^{(2)}\left(b_{1}\right)\right)
\end{aligned}
$$

for all $a_{1}, \ldots, a_{n}, b_{1}, \ldots, b_{n}$ in $\mathscr{B}, t_{1}, \ldots, t_{n}$ in $T$, and for all $n$.

Proof. Assume that (1.1) holds; then the map $U$ given by $U\left(\pi^{(1)} j_{t_{n}}^{(1)}\left(b_{n}\right) \cdots\right.$ 
$\left.\pi^{(1)} j_{t_{1}}^{(1)}\left(b_{1}\right) \Omega^{(1)}\right)=\pi^{(2)} j_{t_{n}}^{(2)}\left(b_{n}\right) \cdots \pi^{(2)} j_{t_{1}}^{(2)}\left(b_{1}\right) \Omega^{(2)}$ extends to a unitary map of $\mathscr{H}^{(1)}$ onto $\mathscr{H}^{(2)}$ such that $U \Omega^{(1)}=\Omega^{(2)}$ and $U \pi^{(1)} j_{t}^{(1)}(b)=\pi^{(2)} j_{t}^{(2)}(b) U$ for all $b$ in $\mathscr{B}$ and $t$ in $T$. The converse is clear.

A stochastic process is said to be a $W^{*}$-stochastic process if $\mathscr{B}$ is a $W^{*}$ algebra and the maps $\pi \circ j_{t}$ are normal for all $t$ in $T$.

1.2. Let $\mathbb{T}=\cup_{n \in N} T^{n}$ be the set of all $n$-tuples of elements of $T, n=1,2, \ldots$; an element $\mathfrak{t}$ of $\mathbb{T}$ is in $T^{n}$ for some $n=n(\mathfrak{t})$ and can be written $\mathbb{t}=\left(t_{1}, \ldots, t_{n}\right)$. For each $\mathfrak{t}$ in $\mathbb{T}$, let $\mathscr{B}_{\mathfrak{t}}$ be the $n(\mathfrak{t})$-fold Cartesian product of $\mathscr{B}$ and denote an element of $\mathscr{B}_{\mathfrak{t}}$ by $\mathbb{b}=\left(b_{1}, \ldots, b_{n}\right)$, and let $j_{\mathfrak{t}}$ be the map of $\mathscr{B}_{\mathfrak{t}}$ into $\mathscr{A}$ given by $j_{t}(b)=j_{t_{n}}\left(b_{n}\right) \cdots j_{t_{1}}\left(b_{1}\right)$; the correlation kernel $W_{t}$ of the stochastic process $\left(\mathscr{A},\left\{j_{t}\right\}, \omega\right)$ over $\mathscr{B}$ indexed by $T$ is the function on $\mathscr{B}_{\mathfrak{t}} \times \mathscr{B}_{\mathfrak{t}}$ with values in $\mathbb{G}$ given by

$$
w_{\mathfrak{s}}(\mathbf{a} ; \mathbf{b})=\omega\left(j_{\mathfrak{t}}(\mathbf{a}) * j_{\mathfrak{s}}(\mathbf{b})\right) .
$$

According to Proposition 1.1, a stochastic process is determined up to equivalence by the family $\left\{W_{\mathbf{t}}: \mathfrak{t} \in \mathbb{T}\right\}$ of its correlation kernels.

Proposition 1.2. The family $\left\{W_{\mathfrak{t}}(\cdot, \cdot): \mathbb{t} \in \mathbb{T}\right\}$ of correlation kernels of a stochastic process over $\mathscr{B}$ indexed by $T$ satisfies conditions $C K 1, \ldots, C K 6$ below. For a $W^{*}$-process, condition NCK also holds.

CK1 (projectivity): For all $\mathbb{t}$ in $\mathbb{T}$, for $k$ such that $1 \leqq k \leqq n(\mathbb{t})$, and for $\mathbf{a}, \mathbf{b}$ in $\mathscr{B}_{\mathbf{z}}$ such that $a_{k}=b_{k}=1$, we have

$$
w_{\mathrm{z}}(\mathbf{a} ; \mathbf{b})=w_{\hat{k} \mathrm{t}}(\hat{k} \mathrm{a} ; \hat{k} \mathbf{b})
$$

where $\hat{k} \mathbf{t}=\left(t_{1}, \ldots, t_{k-1}, \hat{t}_{k}, t_{k+1}, \ldots, t_{n}\right), \hat{k} \mathbf{a}=\left(a_{1}, \ldots, \hat{a}_{k}, \ldots, a_{n}\right)$;

CK2 (positivity): For all $\mathbb{t}$ in $\mathbb{T}$, for all finite sequences $\left\{c_{r} \in \mathbb{C}: r=\right.$ $1, \ldots, m\},\left\{\mathbb{b}_{r} \in \mathscr{B}_{\mathfrak{r}}: r=1, \ldots, m\right\}$, we have

$$
\sum_{i, j} \bar{c}_{i} c_{j} w_{t}\left(\mathfrak{b}_{i} ; \mathbb{b}_{j}\right) \geqq 0 ;
$$

CK3 (normalization): For all $\mathbb{t}$ in $\mathbb{T}$, we have

$$
w_{\mathrm{t}}(\mathbb{1} ; \mathbb{1})=1 \text {, }
$$

where $\mathbb{1}$ is the element $(1, \ldots, 1)$ of $\mathscr{B}_{t}$;

CK4 (sesquilinearity): For all $\mathbb{t}$ in $\mathbb{T}$, for all $\mathbb{\mathfrak { a }}$, in $\mathscr{B}_{\mathfrak{t}}$, the map $b_{k} \mapsto$ $w_{t}(\mathbf{a} ; \mathbf{b})$ from $\mathscr{B}$ to $\mathbb{C}$ is linear and the map $a_{k} \mapsto w_{\mathbf{t}}(\mathfrak{a} ; \mathfrak{b})$ is conjugate linear, for each $k$ such that $1 \leqq k \leqq n(t)$; 
CK5 (*-condition): For all $\mathbf{t}$ in $\mathbf{T}$ and for $n=n(\mathbf{t})$, the map $a_{n}, b_{n} \mapsto$ $w_{\mathbf{t}}(\mathbf{a} ; \mathbf{b})$ of $\mathscr{B} \times \mathscr{B}$ to $\boldsymbol{C}$ factors through the map $a_{n}, b_{n} \mapsto a_{n}^{*} b_{n}$ of $\mathscr{B} \times \mathscr{B}$ to $\mathscr{B}$;

CK6 (multiplicativity): For all $\mathbf{t}$ in $\mathbf{T}$ such that $t_{k}=t_{k-1}$ we have

$$
w_{\mathbf{t}}(\mathbf{a} ; \mathbf{b})=w_{\hat{k} \mathbf{t}}(k \mathbf{a} ; k \mathbf{b})
$$

for all $\mathbf{a}, \mathbf{b}$ in $\mathscr{B}_{\mathbf{z}}$, where $k \mathbf{a}=\left(a_{1}, \ldots, a_{k} a_{k-1}, a_{k+1}, \ldots, a_{n}\right)$ is in $\mathscr{B}_{\hat{k} \mathbf{t}}$ for $\mathbf{a}=$ $\left(a_{1}, \ldots, a_{k-1}, a_{k}, a_{k+1}, \ldots, a_{n}\right)$ in $\mathscr{B}_{\mathrm{t}}$;

NCK (normality): For all $\mathbf{t}$ in $\mathbf{T}$, all a in $\mathscr{B}_{\mathbf{t}}$ such that $a_{n}=1, n=n(\mathbf{t})$, and for all $\mathbf{b}$ in $\mathscr{B}_{\mathbf{t}}$, the map $b_{n} \mapsto w_{\mathbf{t}}(\mathbf{a} ; \mathbf{b})$ of $\mathscr{B}$ to $\boldsymbol{C}$ is a normal map.

Proof. A straightforward verification.

1.3. Let $\mathscr{B}$ be a $C^{*}$-algebra with identity and $T$ a set: a projective system of correlation kernels over $\mathscr{B}$, indexed by $T$, is a family $\left\{w_{\mathbf{z}}: \mathbf{t} \in \mathbf{T}\right\}$ of functions $w_{\mathrm{t}}: \mathscr{B}_{\mathrm{t}} \times \mathscr{B}_{\mathrm{t}} \rightarrow C$ such that conditions $C K 1$ to $C K 6$ hold. If, in addition, $\mathscr{B}$ is a $W^{*}$-algebra and condition $N C K$ holds, then the family is said to be a projective system of normal correlation kernels. Notice that, for all $\mathbf{t}$ in $\mathbf{T}$, b in $\mathscr{B}_{\mathfrak{t}}$, and $\mathbf{a}=\left(b_{1}, \ldots, b_{n-1}, 1\right)$ in $\mathscr{B}_{\mathbf{t}}$, the map $b_{n} \mapsto w_{\mathbf{t}}(\mathbf{a} ; \mathbf{b})$ is a positive linear functional on $\mathscr{B}$ by $C K 2, C K 4$ and $C K 5$; hence it is bounded.

Theorem 1.3 (Reconstruction Theorem). Let $\mathscr{B}$ be a $C^{*}$-algebra with identity, and let $\left\{w_{\mathbf{t}}: \mathbf{t} \in \mathbf{T}\right\}$ be a projective system of correlation kernels over $\mathscr{B}$, indexed by $T$; then there exists a stochastic process $\left(\mathscr{A},\left\{j_{t}: t \in T\right\}, \omega\right)$ over $\mathscr{B}$, indexed by $T$, having $\left\{w_{\mathbf{s}}: \mathbf{t} \in \mathbf{T}\right\}$ as its family of correlation kernels; the process is unique up to equivalence. Moreover, if $\mathscr{B}$ is a $W^{*}$-algebra and $\left\{w_{\mathbf{z}}: \mathbf{t} \in \mathbf{T}\right\}$ satisfies the NCK condition, then the process is a $W^{*}$-process.

Proof. We realize $\mathscr{A}$ as a concrete $C^{*}$-algebra of operators on a Hilbert space $\mathscr{H}$ with cyclic vector $\Omega$; we use $C K 1, C K 2$ and $C K 3$ to consruct $\mathscr{H}$ and $\Omega$, and $C K 4, C K 5$ and $C K 6$ to construct the maps $j_{t}$ and the algebra $\mathscr{A}$. First, we construct a set $X$ containing all the $\mathscr{B}_{\mathbf{t}}$ and a positive-definite kernel $w$ on $X \times X$. We define a partial ordering $\prec$ in $\mathbf{T}:$ put $\mathbf{s} \prec \mathbf{t}$ if $n(\mathbf{s}) \leqq n(\mathbf{t})$ and $\mathbf{s}$ can be obtained from $\mathbf{t}$ by deleting components of $\mathbf{t}=\left(t_{1}, \ldots, t_{n}\right)$. Whenever $\mathbf{s} \prec \mathbf{t}$ and $\mathbf{s}=\left(s_{1}, \ldots, s_{m}\right), \mathbf{t}=\left(t_{1}, \ldots, t_{n}\right)$ define $\kappa_{s, \mathbf{t}}$ to be the function from $\{1, \ldots, m\}$ to $\{1, \ldots, n\}$ defined by

$$
\begin{aligned}
& \kappa_{\mathbf{s}, \mathbf{t}}(m)=\max \left\{l ; t_{l}=s_{m}\right\} \\
& \kappa_{\mathbf{s}, \mathbf{t}}(r)=\max \left\{l: l<\kappa_{\mathbf{s}, \mathbf{t}}(r+1), t_{l}=s_{r}\right\}, r<m,
\end{aligned}
$$

and let $f_{\mathrm{a}}$ be the embedding of $\mathscr{B}_{\mathrm{s}}$ into $\mathscr{B}_{\mathbf{z}}$ given by 


$$
\begin{aligned}
& f_{\mathbf{s}}^{\mathbf{z}}(\mathbf{a})=\left(b_{1}, \ldots, b_{n}\right), \\
& b_{l}= \begin{cases}1 & \text { if } \quad l \neq \kappa_{\mathbf{s}, \mathrm{z}}(r) \text { for all } r=1, \ldots, m, \\
a_{r} & \text { if } \quad l=\kappa_{\mathrm{s}, \mathrm{z}}(r) .\end{cases}
\end{aligned}
$$

Then $f_{\mathfrak{t}}^{\mathfrak{z}}$ is the identity map on $\mathscr{B}_{\mathfrak{s}}$, and $f_{\mathfrak{z}}^{\mathfrak{u}} \circ f_{\mathfrak{s}}^{\mathfrak{q}}=f_{\mathfrak{s}}^{\mathfrak{u}}$ whenever $\mathrm{s}<\mathfrak{t}<\mathbb{u}$. Let $X$ be the inductive limit $\lim \left\{\mathscr{B}_{\mathrm{s}}, f_{\mathrm{s}}^{\mathrm{t}}: \mathbf{s} \prec \mathbf{t}\right\}$ in the category of sets; then there exists maps $i_{\mathrm{s}}: \mathscr{B}_{\mathrm{t}} \rightarrow X$ such that $i_{\mathrm{t}} \circ f_{\mathrm{a}}^{\mathrm{t}}=i_{\mathrm{s}}$ for $\mathrm{s}<\mathrm{t}$. By $C K 1$ we have $w_{\mathrm{s}}(\mathbf{a} ; \mathbf{b})$ $=w_{\mathbf{z}}\left(f_{\mathbf{a}}^{\mathbf{t}} \mathbf{a} ; f_{\mathbf{z}}^{\mathbf{t}} \mathbf{b}\right)$ for all $\mathbf{s}<\mathbf{t}$, and $\mathbf{a}, \mathbf{b}$ in $\mathscr{B}_{\mathbf{B}}$; hence there exists a kernel $w$ on $X \times X$ given by $w\left(i_{\mathbf{t}} \mathbf{a} ; i_{\mathbf{t}} \mathbf{b}\right)=w_{\mathbf{t}}(\mathbf{a} ; \mathbf{b})$ for all $\mathbf{a}, \mathbf{b}$ in $\mathscr{B}_{\mathbf{t}}$ and for all $\mathbf{t}$ in $\mathbb{T}$, and by $C K 2$ it is positive-definite. Then, by Theorem 1.9 of [13], there exists a minimal Kolmogorov decomposition $(\mathscr{H}, v)$ of $w$ so that $v: X \rightarrow \mathscr{H},\langle v(x)$, $v(y)\rangle=w(x, y)$ for all $x, y$ in $X$, and $\mathscr{H}=\vee\{v(x): x \in X\}$.

Let $\mathscr{D}$ denote the linear span of $\{v(x): x \in X\}$; it is dense in $\mathscr{H}$ and its elements are of the form $v\left(i_{\mathbf{t}}(\mathbf{b})\right)$ for some $\mathbf{t}$ in $\mathbb{T}$ and $\mathbf{b}$ in $\mathscr{B}_{\mathbf{t}}$. By $C K 1$, the vector $v\left(i_{\mathbf{t}}(\mathbf{1})\right)$ is independent of $\mathbf{t}$ in $\mathbf{T}$; denote the common value by $\Omega$; then $\langle\Omega, \Omega\rangle=1$ by $C K 3$. For all $b$ in $\mathscr{B}$ and $t$ in $T$, define the linear operator $j_{t}(b)$ mapping $\mathscr{D}$ into itself by $j_{t}(b) v\left(i_{\mathbf{s}}(\mathbf{a})\right)=v\left(i_{\mathbf{s}, t}(\mathbf{a}, b)\right)$ where $\mathbf{s}, t=\left(s_{1}, \ldots, s_{m}, t\right)$ and $\mathbf{a}, b=$ $\left(a_{1}, \ldots, a_{m}, b\right)$. We have

$$
v\left(i_{\mathbf{t}}(\mathbf{b})\right)=j_{\mathbf{t}}(\mathbf{b}) \Omega \quad \text { for all } \mathfrak{t} \text { in } \mathbf{T} \text { and } \mathbf{b} \text { in } \mathscr{B}_{\mathbf{t}},
$$

and $j_{t}(1)$ is the identity map of $\mathscr{D}$ by $C K 1$. So far, only conditions $C K 1, C K 2$, $C K 3$ have been used. Now we use $C K 2, C K 4$ and $C K 5$ to conclude that

$$
\left\|j_{t}(b) v\left(i_{\mathbf{s}}(\mathbf{a})\right)\right\|^{2}=w_{\mathbf{B}, t}(\mathbf{a}, b ; \mathbf{a}, b) \leqq\|b\|^{2} w_{\mathbf{s}}(\mathbf{a} ; \mathbf{a})=\|b\|^{2}\left\|v\left(i_{\mathbf{s}}(\mathbf{a})\right)\right\|^{2}
$$

so that $j_{t}(b)$ extends by linearity and continuity to a bounded linear operator $j_{t}(b)$ on $\mathscr{H}$ with $\left\|j_{t}(b)\right\| \leqq\|b\|$. From $C K 4, C K 5, C K 6$ it follows that $j_{t}$ is a $*$-representation of $\mathscr{B}$ for each $t$ in $T$. Let $\mathscr{A}$ be the $C^{*}$-algebra generated by $\left\{j_{t}(b): b \in \mathscr{B}, t \in T\right\}$ and let $\omega$ bc the vector state on $\mathscr{A}$ given by $\omega(a)=\langle\Omega, a \Omega\rangle$ for all $a$ in $\mathscr{A}$. Then, by (1.3), it follows that $\Omega$ is cyclic for $\mathscr{A}$ in $\mathscr{H}$ and $\left(\mathscr{A},\left\{j_{t}: t \in T\right\}, \omega\right)$ is a stochastic process over $\mathscr{B}$ satisfying

$$
\omega\left(j_{\mathfrak{s}}(\mathbf{a})^{*} j_{\mathbf{s}}(\mathbf{b})\right)=w_{\mathbf{t}}(\mathbf{a} ; \mathbf{b}) \text { for all } \mathbb{t} \text { in } \mathbb{T} \text { and } \mathbf{a}, \mathbf{b} \text { in } \mathscr{B}_{\mathbf{s}} \text {. }
$$

By Proposition 1.1, the process is unique up to equivalence. It is clear from the construction that condition $N C K$ ensures that the maps $\pi \circ j_{t}$ are normal.

1.4. Let $\left(\mathscr{A},\left\{j_{t}\right\}, \omega\right)$ be a stochastic process over $\mathscr{B}$, indexed by $T$, and let $G$ be a group which acts on $T$; the process is $G$-stationary if there is a group $\left\{U_{g}: g \in G\right\}$ of unitaries on the GNS space $\mathscr{H}$ of $(\mathscr{A}, \omega)$ such that 


$$
S: U_{g} \Omega=\Omega \quad \text { and } \quad U_{g} \pi j_{t}(b)=\pi j_{g t}(b) U_{g}
$$

for all $g$ in $G$ and $b$ in $\mathscr{B}$. Define an action of $G$ on $\mathbb{T}$ by $g\left(t_{1}, \ldots, t_{n}\right)=$ $\left(g t_{1}, \ldots, g t_{n}\right)$. A family $\left\{w_{\mathbf{t}}: \mathfrak{t} \in \mathbb{T}\right\}$ of correlation kernels is said to be $G$ invariant if we have

$$
S^{\prime}: w_{g \mathbf{t}}(\mathbf{a} ; \mathbf{b})=w_{\mathbf{t}}(\mathbf{a} ; \mathbf{b})
$$

for all $g$ in $G, \mathfrak{t}$ in $\mathbb{T}$, and $\mathbf{a}, \mathbb{b}$ in $\mathscr{B}_{\mathfrak{t}}$.

Proposition 1.4. A stochastic process is G-stationary if and only if its family of correlation kernels is G-invariant.

Proof. Condition $S^{\prime}$ implies that the processes $\left(\mathscr{A},\left\{j_{t}\right\}, \omega\right)$ and $(\mathscr{A}$, $\left.\left\{j_{g t}\right\}, \omega\right)$ are equivalent: the existence of unitaries satisfying $S$ and the group property then follows from Proposition 1.1. The converse implication is clear.

When a process is $G$-stationary with $G=T=\mathbb{R}$ and the action of $\mathbb{R}$ on $\mathbb{R}$ given by $s, t \mapsto s+t$, we shall simply say that the process is stationary.

1.5. Let $S_{n}$ denote the symmetric group on $n$ objects; define the action of $S_{n}$ on $T^{n}$ by $\mathbf{t} \mapsto p \mathbf{t}$ with $p\left(t_{1}, \ldots, t_{n}\right)=\left(t_{p(1)}, \ldots, t_{p(n)}\right)$ and on $\mathscr{B}_{\mathbf{t}}$ by $\mathbf{b} \mapsto p \mathbf{b}$ with $p\left(b_{1}, \ldots, b_{n}\right)=\left(b_{p(1)}, \ldots, b_{p(n)}\right)$. A family $\left\{w_{\mathbf{t}}: \mathbf{t} \in \mathbb{T}\right\}$ of correlation kernels is said to be symmetric if we have $w_{p \mathbf{t}}(p \mathbf{a} ; p \mathbf{b})=w_{\mathbf{t}}(\mathbf{a} ; \mathbf{b})$ for all $\mathbb{t}$ in $\mathbb{T}$, all $\mathbf{a}, \mathbf{b}$ in $\mathscr{B}_{\mathbf{t}}$ and all $p$ in $S_{n(\mathbf{t})}$, such that $p(k)<p(l)$ if $k<l$ and $t_{k}=t_{l}$; the family is said to be totally symmetric if $w_{p \mathbf{t}}(p \mathbf{a} ; p \mathbf{b})=w_{\mathbf{t}}(\mathbf{a} ; \mathbf{b})$ holds for all $p$ in $S_{n(\mathbf{t})}$ without restriction. A stochastic process is said to be (totally) symmetric if the family of its correlation kernels is (totally) symmetric.

Proposition 1.5. A stochastic process is symmetric if and only if the algebras $\pi j_{t}(\mathscr{B})$ and $\pi j_{s}(\mathscr{B})$ at distinct times commute: $\left[\pi j_{s}(a), \pi j_{t}(b)\right]=0$ for all $a, b$ in $\mathscr{B}$ whenever $s \neq t$; it is totally symmetric if and only if the algebras at distinct times commute and the algebras $\pi j_{t}(\mathscr{B})$ at fixed times are abelian. (The proof is straightforward.)

\section{Commentary on Section 1 and Supplementary Results}

1.6. The definition of stochastic process given in Section 1.1 was framed with the quantum theory of open systems in mind; it may be thought of as a non-commutative version of a classical stochastic process in the sense of Doob [1] and of Meyer [2]. A classical stochastic process with values in a measurable space $(S, \mathscr{E})$, indexed by a set $T$, is determined by a family $\left\{X_{t}: t \in T\right\}$ of measurable functions defined on a probability space $(\Sigma, \mathscr{F}, \mu)$ with values in $S$; 
two processes indexed by the same set, and with values in the same space, are said to be equivalent if they have the same finite-dimensional joint distributions:

$$
\int_{\Sigma(1)} f_{1} \circ X_{t_{1}}^{(1)} \cdots f_{n} \circ X_{t_{n}}^{(1)} d \mu^{(1)}=\int_{\Sigma(2)} f_{1} \circ X_{t_{1}}^{(2)} \cdots f_{n} \circ X_{t_{n}}^{(2)} d \mu^{(2)}
$$

for each finite sequence $\left\{f_{1}, \ldots, f_{n}\right\}$ in a set of generators of the $W^{*}$-algebra $L^{\infty}(S, \mathscr{E})$, and each finite sequence $\left\{t_{1}, \ldots, t_{n}\right\}$ of elements of $T$. Each $X_{t}$ determines a normal identity-preserving *-homomorphism $j_{t}$ of $\mathbb{L}^{\infty}(S, \mathscr{E})$ into $L^{\infty}(\Sigma, \mathscr{F})$ by $j_{t}(f)=f \circ X_{t}$ for each $f$ in $L^{\infty}(S, \mathscr{E})$, the probability measure $\mu$ determines a normal state $\omega$ on $L^{\infty}(\Sigma, \mathscr{F})$ by $\omega(g)=\int_{\Sigma} g d \mu$ for each $g$ in $L^{\infty}(\Sigma, \mathscr{F})$, and the GNS representation induced by $\omega$ is the quotient map of $L^{\infty}(\Sigma, \mathscr{F})$ onto $L^{\infty}(\Sigma, \mathscr{F}, \mu)$. Thus a classical stochastic process in the sense of Doob defines a $W^{*}$-stochastic process $\left(\mathscr{A},\left\{j_{t}\right\}, \omega\right)$ over an abelian $W^{*}$ algebra $\mathscr{B}$, with $\mathscr{A}$ abelian.

Conversely, let $\left(\mathscr{A},\left\{j_{t}\right\}, \omega\right)$ be a $W^{*}$-stochastic process over an abelian algebra $\mathscr{B}$ with $\pi(\mathscr{A})$ abelian; then, by the representation theorem for abelian $W^{*}$-algebras [14] there exists a measurable space $(S, \mathscr{E})$ and a probability space $(\Sigma, \mathscr{F}, \mu)$ such that $\mathscr{B}=L^{\infty}(S, \mathscr{E})$ and $\pi(\mathscr{A})^{\prime \prime}=L^{\infty}(\Sigma, \mathscr{F}, \mu)$, the state $\omega$ is determined by the probability measure $\mu$, and there exists a family $\left\{X_{t}: t \in T\right\}$ of measurable functions $X_{t}: \Sigma \rightarrow S$ such that $j_{t}(f)=f \circ X_{t}$ for each $f$ in $\mathscr{B}$ (cf. Lemma 2.3 of [3]). In this case, the equivalence condition of Section 1.1 becomes the equality of finite-dimensional joint-distributions; thus we can define a classical stochastic process to be a $W^{*}$-stochastic process for which $\mathscr{B}$ and $\pi(\mathscr{A})$ are abelian.

A stochastic process $\left(\mathscr{A},\left\{j_{t}\right\}, \omega\right)$ determines a family of local algebras whose union generates $\mathscr{A}$; for processes in the sense of Doob and their non-commutative versions, local algebras $\mathscr{A}_{F}$ can be associated to finite subsets $F$ of the index set $T$, by defining $\mathscr{A}_{F}$ to be the $C^{*}$-algebra generated by $\left\{j_{t}(b): b \in \mathscr{B}, t \in F\right\}$. Also generalized stochastic processes in the sense of Gelfand [15] and local field theories in the sense of Haag and Kastler [4] give examples of stochastic processes when the index set is chosen and the local algebras are defined appropriately; in these cases, it might be useful to regard two stochastic processes as equivalent if there is a unitary $U$ of $\mathscr{H}^{(1)}$ onto $\mathscr{H}^{(2)}$ such that $U \Omega^{(1)}=\Omega^{(2)}$ and $U \pi^{(1)}\left(\mathscr{A}_{F}^{(1)}\right)^{\prime \prime}=\pi^{(2)}\left(\mathscr{A}_{F}^{(2)}\right)^{\prime \prime} U$ for all local algebras $\mathscr{A}_{F}$.

When applied to stochastic processes in the sense of Doob, such a definition provides a classification of stochastic processes which is too coarse for our 
purposes. It would imply that two stochastic processes $\left(\mathscr{A}^{(i)},\left\{j_{t}^{(i)}\right\}, \omega^{(i)}\right)$, $i=1,2$, over $\mathscr{B}$ are equivalent if there exists a family $\left\{\alpha_{t}: t \in T\right\}$ of $*_{\text {-automor- }}$ phisms of $\mathscr{B}$ such that

$$
\begin{gathered}
\omega^{(1)}\left(j_{t_{1}}^{(1)}\left(a_{1}\right)^{*} \cdots j_{t_{n}}^{(1)}\left(a_{n}\right)^{*} j_{t_{n}}^{(1)}\left(b_{n}\right) \cdots j_{t_{1}}^{(1)}\left(b_{1}\right)\right)=\omega^{(2)}\left(j_{t_{1}}^{(2)}\left(\alpha_{t_{1}} a_{1}\right)^{*}\right. \\
\left.\cdots j_{t_{n}}^{(2)}\left(\alpha_{t_{n}} a_{n}\right)^{*} j_{t_{n}}^{(2)}\left(\alpha_{t_{n}} b_{n}\right) \cdots j_{t_{1}}^{(2)}\left(\alpha_{t_{1}} b_{1}\right)\right)
\end{gathered}
$$

for all $a_{1}, \ldots, a_{n}, b_{1}, \ldots, b_{n}$ in $\mathscr{B}$, and all $t_{1}, \ldots, t_{n}$ in $T$. For example, let $\left\{T_{t}^{(i)}: t \in T\right\}, i=1,2$, be two groups of measurable maps of a measurable space $(S, \mathscr{E})$ into itself, define $\tau_{t}^{(i)}: \mathscr{B}=L^{\infty}(S, \mathscr{E}) \rightarrow \mathscr{B}$ by $\tau_{t}^{(i)}(f)=f \circ T_{t}^{(i)}, \mathscr{A}^{(1)}=$ $\mathscr{A}^{(2)}=\otimes_{\mathbf{R}} \mathscr{B}, j_{t}^{(1)}=j_{t}^{(2)}$ the natural embedding of $\mathscr{B}$ as the $t$-th factor, for each $t$ in $\boldsymbol{R}$. Let $v$ be a probability measure on $(S, \mathscr{E})$ and define states $\omega^{(i)}$ on $\mathscr{A}^{(i)}$ by

$$
\begin{aligned}
\omega^{(i)}\left(j_{t_{1}}^{(i)}\left(a_{1}\right)^{*}\right. & \left.\cdots j_{t_{n}}^{(i)}\left(a_{n}\right)^{*} j_{t_{n}}^{(i)}\left(b_{n}\right) \cdots j_{t_{1}}^{(i)}\left(b_{1}\right)\right) \\
& =v\left(\tau_{t_{1}}^{(i)}\left(a_{1}\right)^{*} \cdots \tau_{t_{n}}^{(i)}\left(a_{n}\right)^{*} \tau_{t_{n}}^{(i)}\left(b_{n}\right) \cdots \tau_{t_{1}}^{(i)}\left(b_{1}\right)\right), i=1,2
\end{aligned}
$$

then the processes $\left(\mathscr{A}^{(i)},\left\{j_{t}^{(i)}\right\}, \omega^{(i)}\right)$ would be equivalent, with $\alpha_{t}$ given by $\tau_{-t}^{(2)} \tau_{t}^{(1)}$ for each $t$ in $\boldsymbol{R}$.

The correlation kernels defined in Section 1.2 are determined by their values $w_{\mathbf{t}}(\mathbf{b} ; \mathbf{b})$ on the diagonal of $\mathscr{B}_{\mathbf{s}} \times \mathscr{B}_{\mathbf{t}}$; these are positive numbers and can, in principle, be determined by sequences of measurement operations $[4,5,6]$; if the set $T$ of indices represents time, then only the correlation kernels with $t_{1} \leqq \cdots \leqq t_{n}$ will be accessible to measurement, but those at arbitrary times can be deduced from the time-ordered ones when the commutation relations among observables at different times are known. From the mathematical point of view, correlation kernels provide a substitute for the finite-dimensional joint distributions of classical probability theory (joint distributions do not exist for non-commuting observables in quantum theory [16]), which are the basic objects in the well-known theorem of Kolmogorov [7], which provides the reconstruction of a classical stochastic process given a projective family of finite-dimensional joint distributions. Our Theorem 1.3 is a non-commutative version, which shows that a stochastic process can be reconstructed from a projective family of correlation kernels.

In the classical case, the Kolmogorov construction does more: it shows that every classical stochastic process over a given algebra $\mathscr{B}=L^{\infty}(S, \mathscr{E})$ and indexed by a given set $T$ can be realized by giving a state $\bar{\omega}$ on a universal algebra $\overline{\mathscr{A}}$, with universal embeddings $\bar{j}_{t}$ of $\mathscr{B}$ into $\overline{\mathscr{A}}$ in such a way that all relations between the image algebras $\overline{\mathscr{A}}_{s}$ and $\overline{\mathscr{A}}_{t}$ for $s \neq t$ are given by the state alone; we 
state below (Theorem 1.7.1) a non-commutative version of this aspect of Kolmogorov's theorem. In the classical case, the universal $\overline{\mathscr{A}}$ can be taken to be $L^{\infty}\left(\Pi_{T} S, \Pi_{T} \mathscr{E}\right)$ and the analytical content of Kolmogorov's theorem asserts that, under weak topological assumptions on $S, \bar{\omega}$ induces a countably additive measure on $\Pi_{T}(S, \mathscr{E})$; in other words, it gives a criterion for the normality of the limit of a net of locally normal states. We shall not discuss here a non-commutative version of this aspect of the theorem.

The first part of the proof of the reconstruction theorem, using little more than Theorem 1.9 of [13] and the inductive limit in the category of sets, is very general: it applies whenever $\mathscr{B}$ is a set with a distinguished element denoted by 1. In a sense it is the common part of all reconstruction theorcms; for instance, Wightman's reconstruction theorem [17] corresponds to the case in which $T$ is the test-function space $\mathscr{S}\left(\boldsymbol{R}^{4}\right)$ and $\mathscr{B}$ is the (non-normed) algebra of polynomials in a single indeterminate $\varphi$ : then $j_{f}(1)=1$ and $j_{f}\left(\varphi^{n}\right)=\varphi(f)^{n}$ for each $f$ in $\mathscr{S}\left(\boldsymbol{R}^{4}\right)$; of course, there is additional structure related to Poincaré invariance and local commutativity which is embodied in the additional Wightman axioms. The Dubin-Sewell result [18] may be viewed as a reconstruction theorem and proved by this procedure: there $\mathscr{B}$ is a normed *algebra with identity which is not closed (being the union of all local $C^{*}$-algebras), $T=\mathbb{R}$ is the time-axis; the additional axioms in this case ensure that the process is stationary and deterministic: $j_{t}(\mathscr{B})^{\prime \prime}=j_{0}(\mathscr{B})^{\prime \prime}$ for all $t$ in $\boldsymbol{R}$.

1.7. If $\mathscr{A}^{(0)}$ is a $C^{*}$-algebra and $\left\{j_{t}^{(0)}: t \in T\right\}$ is a family of identity preserving *-homomorphisms of $\mathscr{B}$ into $\mathscr{A}^{(0)}$, it can be shown that a projective family $\left\{w_{\mathbf{t}}: \mathbf{t} \in \mathbf{T}\right\}$ of correlation kernels over $\mathscr{B}$ defines a state on $\mathscr{A}^{(0)}$ if and only if the algebraic relations of $\mathscr{A}^{(0)}$ are respected by $\left\{w_{\mathfrak{t}}: \mathbb{t} \in \mathbb{T}\right\}$. More precisely, let $\left(\mathscr{A},\left\{j_{t}\right\}, \omega\right)$ be the stochastic process over $\mathscr{B}$ determined by $\left\{w_{\mathbf{t}}: \mathbf{t} \in \mathbb{T}\right\}$ through the reconstruction theorem; then there exists a state $\omega^{(0)}$ on $\mathscr{A}^{(0)}$ such that

$$
\omega^{(0)}\left(j_{\mathbf{t}}^{(0)}(\mathbf{a})^{*} j_{\mathbf{t}}^{(0)}(\mathbf{b})\right)=w_{\mathbf{t}}(\mathbf{a} ; \mathbf{b}) \text { for all } \mathbf{a}, \mathbf{b} \text { in } \mathscr{B}_{\mathbf{s}}, \mathfrak{t} \text { in } \mathbb{T},
$$

if and only if

$$
\sum_{k} j_{\mathbf{t}_{k}}^{(0)}\left(\mathbf{b}_{k}\right)=0 \quad \text { implies } \quad \sum_{k} j_{\mathbf{t}_{k}}\left(\mathbf{b}_{k}\right)=0
$$

for all finite sequences $\left\{\mathbf{t}_{k}: \mathbf{t}_{k} \in \mathbf{T}, k=1, \ldots, m\right\}$ and $\left\{\mathbf{b}_{k}: \mathbf{b}_{k} \in \mathscr{B}_{\mathbf{t}_{k}}, k=1, \ldots, m\right\}$. In particular, if $\left(\mathscr{A}^{(0)},\left\{j_{t}^{(0)}\right\}, \omega^{(0)}\right)$ is a stochastic process, then the process $\left(\mathscr{A},\left\{j_{t}\right\}, \omega\right)$ obtained through the reconstruction theorem is such that $\mathscr{A}$ is 
isomorphic to $\pi^{(0)}\left(\mathscr{A}^{(0)}\right)$; so all algebraic relations of $\mathscr{A}^{(0)}$ are preserved in $\mathscr{A}$, and further-statistical-relations may be introduced by the state $\omega^{(0)}$, if $\pi^{(0)}$ is not faithful.

We state without proof two theorems on the existence of a universal $C^{*}$ algebra for all stochastic processes (respectively, for all symmetric stochastic processes) over a given $C^{*}$-algebra $\mathscr{B}$, indexed by a set $T$; they are made plausible by the considerations above. The proof of Theorem 1.7.1 is the same as in Winnink [19].

Theorem 1.7.1. Let $\mathscr{B}$ be a $C^{*}$-algebra with identity, and $T$ a set. There exists a $C^{*}$-algebra with identity $\overline{\mathscr{A}}$ and a family $\left\{j_{t}: t \in T\right\}$ of $*_{\text {-homomor- }}$ phisms of $\mathscr{B}$ into $\overline{\mathscr{A}}$ such that any stochastic process $\left(\mathscr{A},\left\{j_{t}\right\}, \omega\right)$ over $\mathscr{B}$ indexed by $T$ is equivalent to a stochastic process $\left(\overline{\mathscr{A}},\left\{\bar{j}_{t}\right\}, \bar{\omega}\right)$ where only $\bar{\omega}$ depends on $\left(\mathscr{A},\left\{j_{t}\right\}, \omega\right)$.

Theorem 1.7.2. A stochastic process $\left(\mathscr{A},\left\{j_{t}\right\}, \omega\right)$ over $\mathscr{B}$ indexed by $T$ is symmetric if and only if it is equivalent to a stochastic process $\left(\otimes_{T} \mathscr{B}\right.$, $\left.\left\{i_{t}\right\}, \bar{\omega}\right)$, where $\otimes_{T} \mathscr{B}$ is a $C^{*}$-tensor product of isomorphic copies of $\mathscr{B}$ indexed by $t \in T, i_{t}$ is the natural embedding of $\mathscr{B}$ as the $t$-th component of the tensor product, and $\bar{\omega}$ is any state on $\otimes_{T} \mathscr{B}$.

The algebra $\overline{\mathscr{A}}$ of Theorem 1.6 (cf. [19]) is a quotient of the free algebra generated by the disjoint union of isomorphic copies of $\mathscr{B}$ indexed by $t \in T$; the only algebraic relations imposed in $\overline{\mathscr{A}}$ are those following from the requirement that the $\left\{j_{t}: t \in T\right\}$ are *-homomorphisms. The algebra $\otimes_{T} \mathscr{B}$ is obtained from $\overline{\mathscr{A}}$ by quotienting out all commutators between observables localized at different times. It is an analogue of the algebra $L^{\infty}\left(\Pi_{T} S, \Pi_{T}(\mathscr{E})\right)$ of bounded measurable functions on the path space of a classical stochastic process. Of course, all classical processes are symmetric, indeed, totally symmetric. Symmetric quantum stochastic processes over $\mathscr{B}=\mathscr{B}(\mathscr{H})$ for some Hilbert space $\mathscr{H}$ have been studied by Accardi in [3, 20].

1.8. For symmetric processes, the reconstruction theorem can be cast in a form which is more similar to the original theorem of Kolmogorov [7]; first we introduce some suitable notation. Let $F(T)$ denote the collection of finite subsets of $T$; for each $E$ in $F(T)$ let $\mathscr{B}^{E}$ denote the functions from $E$ to $\mathscr{B}$ equipped with the operations of pointwise addition, multiplication and taking of adjoints; for example, for $\beta_{1}$ and $\beta_{2}$ in $\mathscr{B}^{E}$ we have $\left(\beta_{1} \beta_{2}\right)(s)=\beta_{1}(s) \beta_{2}(s)$ for 
all $s$ in $E$. For each $t$ in $T$ define an action of $\mathscr{B}$ on $\mathscr{B}^{E}$ by $\left(b_{t} \beta\right)(t)=b \beta(t)$, $\left(b_{t} \beta\right)(s)=\beta(s)$ for $s \neq t . \quad$ For $E, F$ in $F(T)$ such that $E \subseteq F$ let $g_{E}^{F}$ be the embedding of $\mathscr{B}^{E}$ in $\mathscr{B}^{F}$ defined for $s$ in $F$ by $\left(g_{E}^{F} \beta\right)(s)=\beta(s)$ for $s$ in $E,\left(g_{E}^{F} \beta\right)(s)=1$ otherwise. Let $[\cdot]: \mathbb{T} \rightarrow F(T)$ be the map given by $[\mathfrak{t}]=\left\{t_{1}, \ldots, t_{n}\right\}$ for each $\mathbb{t}=\left(t_{1}, \ldots, t_{n}\right)$ in $\mathbb{T}$, and let $[\cdot]: \mathscr{B}_{\mathfrak{t}} \rightarrow \mathscr{B}^{[\mathrm{t}]}$ be the map given by $[\mathrm{b}](s)=\prod_{\left\{k: t_{k}=s\right\}} b_{k}$.

Let $\left(\mathscr{A},\left\{j_{t}\right\}, \omega\right)$ be a symmetric stochastic process over a $C^{*}$-algebra $\mathscr{B}$, indexed by a set $T$; the family $\left\{\varphi_{E}: E \in F(T)\right\}$ of functionals given for each $\beta$ in $\mathscr{B}^{E}$ by $\varphi_{E}(\beta)=\omega\left(\prod_{s \in E} j_{s}(\beta(s))\right)$ is called the family of expectations functionals of the process.

Proposition 1.8.1. The family $\left\{\varphi_{E}: E \in F(T)\right\}$ of expectation functionals of a symmetric stochastic process over $\mathscr{B}$ satisfies conditions $E F 1, \ldots, E F 4$; if the process is a $W^{*}$-process, condition NEF also holds:

$E F 1$ (projectivity): $\varphi_{F}\left(g_{E}^{F} \beta\right)=\varphi_{E}(\beta)$ for all $E \leqq F$ in $F(T), \beta$ in $\mathscr{B} E$;

EF2 (positivity): For all $E$ in $F(T)$, we have $\varphi_{E}\left(\beta^{*} \beta\right) \geqq 0$ for all $\beta$ in $\mathscr{B}^{E}$;

$E F 3$ (normalization): For all $E$ in $F(T)$, we have $\varphi_{E}\left(1_{E}\right)=1$ where $1_{E}(s)=1$ for all $s$ in $E$;

EF4 (linearity): For all $E$ in $F(T)$ and all $\beta$ in $\mathscr{B}^{E}$, the map $\beta(s) \mapsto$ $\varphi_{E}(\beta)$ is linear for each $s$ in $E$;

$N E F$ (normality): For all $E$ in $F(T)$ and all $\beta$ in $\mathscr{B}^{E}$, and $t$ in $E$, the map $b \mapsto \varphi_{E}\left(b_{t} \beta\right)$ is a normal map.

Proof. A straightforward verification.

Let $\mathscr{B}$ be a $C^{*}$-algebra with identity and $T$ a set; a family $\left\{\varphi_{E}: E \in F(T)\right\}$ is said to be a projective system of expectation functionals over $\mathscr{B}$, indexed by $T$, if for each $E$ in $F(T), \varphi_{E}$ is a functional on $\mathscr{B}^{E}$ with values in $\mathbb{C}$ such that $E F 1$ to EF4 hold; it is said to be a normal projective system if $\mathscr{B}$ is a $W^{*}$ algebra and also $N E F$ holds.

Corollary 1.8.2. Let $\mathscr{B}$ be a $C^{*}$-algebra with identity, and $T$ a set; let $\left\{\varphi_{E}: E \in F(T)\right\}$ be a projective system of expectation functionals over $\mathscr{B}$, indexed by $T$. Then there exists a symmetric stochastic process $\left(\mathscr{A},\left\{j_{t}\right\}, \omega\right)$ over $\mathscr{B}$ indexed by $T$ having $\left\{\varphi_{E}: E \in F(T)\right\}$ as its family of expectation functionals. The process is unique up to equivalence, and is a $W^{*}$-process if $\mathscr{B}$ is $a W^{*}$-algebra and the system of expectation functionals is normal.

Proof. For each $\mathbf{a}, \mathbf{b}$ in $\mathscr{B}_{\mathfrak{t}}, \mathfrak{t}$ in $\mathbb{T}$, put $w_{\mathfrak{t}}(\mathfrak{a} ; \mathbb{b})=\varphi_{[\mathbf{t}]}\left(\left[\mathfrak{a}^{*}\right]\left[\mathfrak{b}^{*}\right]^{*}\right)$, we check that if $\left\{\varphi_{E}: E \in F(T)\right\}$ satisfies $E F 1$ to $E F 4$, then $\left\{w_{\mathfrak{t}}: \mathbb{t} \in \mathbb{T}\right\}$ satisfies 
$C K 1$ to $C K 6$ (and also $N C K$ if $N E F$ holds). Hence, by Theorem 1.3, there exists a stochastic process $\left(\mathscr{A},\left\{j_{t}\right\}, \omega\right)$ over $\mathscr{B}$, indexed by $T$, unique up to equivalence, having $\left\{w_{\mathbf{t}}: \mathbf{t} \in \mathbf{T}\right\}$ as its family of correlation kernels, so that $w_{\mathbf{t}}(\mathbf{a} ; \mathbf{b})=\omega\left(j_{\mathbf{t}}(\mathbf{a})^{*} j_{t}(\mathbf{b})\right)$. But $[p \mathbf{t}]=[\mathbf{t}]$ and $[p \mathbf{b}]=[\mathbf{b}]$ for all permutations $p$, so that the family $\left\{w_{\mathbf{z}}: \mathbf{t} \in \mathbf{T}\right\}$ is symmetric, and

$$
\varphi_{[\mathbf{t}]}([\mathbf{b}])=w_{\mathbf{t}}\left(\left[\mathbf{b}^{*}\right] ; 1_{\mathbf{t}}\right)=\omega\left(j_{\mathbf{t}}\left(\mathbf{b}^{*}\right)^{*}\right)=\omega\left(j_{t_{1}}\left(b_{1}\right), \ldots, j_{t_{n}}\left(b_{n}\right)\right)
$$

for all $\mathbf{b}=\left(b_{1}, \ldots, b_{n}\right)$ in $\mathscr{B}_{\mathbf{t}}, \mathbf{t}=\left(t_{1}, \ldots, t_{n}\right)$ in $\mathbf{T}$, so that the expectation functionals are given by the process. Normality of the maps $\pi j_{t}$ if $N E F$ holds follows as in Theorem 1.3.

\section{§ 2. Markov Processes and Semigroups}

2.1. In this section, "stochastic process" is to be taken to mean a triple $\left(\mathscr{A},\left\{j_{t}\right\}, \omega\right)$ as in the definition, where $\mathscr{A}$ is identified with its $G N S$ representation $\pi(\mathscr{A})$, so that $j_{t}=\pi \circ j_{t}$; this is always possible up to equivalence. If $\mathscr{B}$ is a $W^{*}$-algebra and the maps $j_{t}$ are normal ( $W^{*}$-stochastic process), we shall take $\mathscr{A}$ to be the $W^{*}$-algebra generated by $\left\{j_{t}(b): b \in \mathscr{B}, t \in T\right\} ; \omega$ is a normal state on $\mathscr{A}$. We shall also assume the indexing set $T$ to be totally ordered (for instance $\left.\boldsymbol{N}, \boldsymbol{Z}, \boldsymbol{R}_{+}, \boldsymbol{R}\right)$, and we shall refer to it as to "time". For each $t$ in $T$, we define the following $C^{*}$-subalgebras of $\mathscr{A}\left(W^{*}\right.$-subalgebras, for a $W^{*}$-stochastic process):

$$
\begin{aligned}
& \mathscr{A}_{t]}=\vee\left\{j_{s}(b): b \in \mathscr{B}, s \leqq t\right\} ; \\
& \mathscr{A}_{t}=\vee\left\{j_{t}(b): b \in \mathscr{B}\right\}=j_{t}(\mathscr{B}) ; \\
& \mathscr{A}_{[t}=\vee\left\{j_{u}(b): b \in \mathscr{B}, t \leqq u\right\} ;
\end{aligned}
$$

and for all $t<u$ we define

$$
\mathscr{A}_{[t, u]}=\vee\left\{j_{s}(b): b \in \mathscr{B}, t \leqq s \leqq u\right\},
$$

where $\vee S$ denotes the $C^{*}$-algebra (or the $W^{*}$-algebra) generated by a subset $S$ of $\mathscr{A}$.

If $\mathscr{A}$ is a $W^{*}$-algebra and $\omega$ is a faithful normal state on $\mathscr{A}$, a recent result [21], which extends a well known theorem of Takesaki [22], shows that there exists a family of canonical completely positive identity preserving maps $E_{s, t}$ of $\mathscr{A}_{t]}$ into $\mathscr{A}_{s}, s \prec t \in T$, compatible with $\omega$ in the sense that 


$$
\omega \uparrow \mathscr{A}_{t]}=\omega\left\lceil\mathscr{A}_{s]^{\circ}} E_{s, t}, s<t \in T\right.
$$

they are faithful and normal, and satisfy the chain rule

$$
E_{s, t} E_{t, u}=E_{s, u} ; s<t<u \in T .
$$

Such maps are conditional expectations if and only if each of the algebras $\left\{\mathscr{A}_{s]}: s \in T\right\}$ is globally invariant under the modular automorphism group of $\mathscr{A}$ associated to $\omega$ by the Tomita-Takesaki theory (in particular, when $\mathscr{A}$ is abelian).

Definition. A stochastic process $\left(\mathscr{A},\left\{j_{t}\right\}, \omega\right)$, where $\mathscr{A}$ is a $W^{*}$-algebra and $\omega$ is a faithful normal state on $\mathscr{A}$, is said to be a Markov process if the canonical maps $\left\{E_{s, t}: s<t \in T\right\}$ compatible with $\omega$ satisfy

$$
M: E_{s, t}\left(\mathscr{A}_{[s, t]}\right) \subseteq \mathscr{A}_{s} \quad \text { for all } s<t \text { in } T .
$$

(This definition is a slight modification of the one in $[23,24,3]$ ).

Theorem 2.1. Let $\left(\mathscr{A},\left\{j_{t}\right\}, \omega\right)$ be a Markov process over $\mathscr{B}(\mathscr{B}, \mathscr{A}$ $W^{*}$-algebras, $j_{t}$ normal maps, $\omega$ a faithful normal state on $\left.\mathscr{A}\right)$. Assume the existence of the left inverse $j_{t}^{*}$ of $j_{t}$, and let $E_{t, t}$ be the identity map of $\mathscr{A}_{t]}$. Then

$$
Z_{s, t}=j_{s}^{*} E_{s, t} j_{t}, s \leqq t \in T
$$

defines a two-parameter family of completely positive identity preserving normal maps of $\mathscr{B}$ into itself, satisfying

$$
Z_{s, t} Z_{t, u}=Z_{s, u} \quad \text { for all } s \leqq t \leqq u \text { in } T .
$$

Moreover, if the $E_{s, t}$ are conditional expectations, then

$$
\begin{aligned}
E_{t_{0}, t_{n}}\left(j_{t_{1}}\left(a_{1}\right)^{*} \cdots j_{t_{n}}\left(a_{n}\right)^{*} j_{t_{n}}\left(b_{n}\right) \cdots j_{t_{1}}\left(b_{1}\right)\right) \\
\quad=j_{t_{0}} Z_{t_{0}, t_{1}}\left(a_{1}^{*} Z_{t_{1}, t_{2}}\left(a_{2}^{*} \cdots Z_{t_{n-1}, t_{n}}\left(a_{n}^{*} b_{n}\right) \cdots b_{2}\right) b_{1}\right)
\end{aligned}
$$

for all $t_{0} \leqq t_{1} \leqq \cdots \leqq t_{n}$ in $T, a_{1}, \ldots, a_{n}, b_{1}, \ldots, b_{n}$ in $\mathscr{B}$.

Proof. For all $s \leqq t$ and all $b$ in $\mathscr{B}$, it follows from the Markov condition $M$ that $E_{s, t} j_{t}(b)$ is in $\mathscr{A}_{s}$, so that $j_{s}^{*} E_{s, t} j_{t}(b)$ makes sense, and $Z_{s, t}$ is well-defined by (2.4); it is completely positive, identity preserving and normal, since it is composed of maps having these properties. Using the chain rule (2.3) and the fact that $j_{t} j_{t}^{*}$ is the identity map on $\mathscr{A}_{t}$, we have

$$
Z_{s, t} Z_{t, u}=j_{s}^{*} E_{s, t} j_{t} j_{t}^{*} E_{t, u} j_{u}=j_{s}^{*} E_{s, t} E_{t, u} j_{u}=j_{s}^{*} E_{s, u} j_{u}=Z_{s, u} \quad \text { for } \quad s \leqq t \leqq u
$$

proving (2.5). Again by (2.3), for $t_{0} \leqq t_{1} \leqq \cdots \leqq t_{n}$, we have $E_{t_{0}, t_{n}}=E_{t_{0}, t_{1}} E_{t_{1}, t_{2}} \cdots$ 
$E_{t_{n-1}, t_{n}}$; if the $E_{s, t}$ are conditional expectations, the left hand side of (2.6) becomes

$$
E_{t_{0}, t_{1}}\left(j_{t_{1}}\left(a_{1}\right)^{*} E_{t_{1}, t_{2}}\left(j_{t_{2}}\left(a_{2}\right)^{*} E_{t_{2}, t_{3}}\left(\cdots E_{t_{n-1}, t_{n}}\left(j_{t_{n}}\left(a_{n}^{*} b_{n}\right)\right) \cdots\right) j_{t_{2}}\left(b_{2}\right)\right) j_{t_{1}}\left(b_{1}\right)\right) \text {. }
$$

Then (2.6) follows from (2.4), the Markov property $M$ and the fact that $j_{t}^{*} j_{t}$ is the identity map on $\mathscr{A}_{t}$.

2.2. If the $\left\{E_{s, t}: s<t \in T\right\}$ compatible with $\omega$ are conditional expectations, there is a projective family $\left\{E_{s]}: s \in T\right\}$ of conditional expectations $E_{s]}$ of $\mathscr{A}$ onto $\mathscr{A}_{s]}, s \in T$, compatible with $\omega$, such that $E_{s, t}=E_{s]}\left\lceil\mathscr{A}_{t]}\right.$ for all $s<t$ in $T$; and the Markov property $M$ is equivalent to

$$
M^{\prime}: E_{s]}\left(\mathscr{A}_{[s}\right)=\mathscr{A}_{s} \quad \text { for all } s \text { in } T .
$$

We list some properties of the family $\left\{E_{s]}: s \in T\right\}$ which we are going to use in the following:

$$
\begin{array}{ll}
E 1: E_{s\rfloor}(a b)=E_{s]}(a) b & \text { for all } a \text { in } \mathscr{A}, b \text { in } \mathscr{A}_{s]}, s \text { in } T ; \\
E 2: \omega=\omega\left\lceil\mathscr{A}_{s]} \circ E_{s]}\right. & \text { for all } s \text { in } T ; \\
E 3: E_{s]} E_{t]}=E_{s \wedge t]} & \text { for all } s, t \text { in } T ;
\end{array}
$$

and if $T=\mathbb{R}$ and the process is also stationary, with group of automorphisms $\left\{u_{t}=U_{t}(\cdot) U_{-t}: t \in \mathbb{R}\right\}$,

$$
E 4: u_{t} E_{s]}=E_{s+t]} u_{t} \quad \text { for all } s, t \text { in } \boldsymbol{R} \text {. }
$$

Conditions $E 1-E 4$ can be formulated also when $\mathscr{A}$ is only a $C^{*}$-algebra and $\omega$ is not a faithful state. If maps satisfying $E 1$ and $E 2$ exist, they satisfy also $E 3$ (and E4 in the stationary case) if the GNS representation of $\mathscr{A}_{s]}$ determined by $\omega\left\lceil\mathscr{A}_{s]}\right.$ is faithful for all $s$. We can use conditions $E 1$ to $E 4$ and $M^{\prime}$ to define a version of the Markov property which makes sense also when $\mathscr{A}$ is not a $W^{*}$-algebra and $\omega$ is not faithful.

Definition. A stochastic process $\left(\mathscr{A},\left\{j_{t}\right\}, \omega\right)$ is said to be a Markov process with conditional expectations if there exists a family $\left\{E_{s]}: s \in T\right\}$ of conditional expectations $E_{s]}$ of $\mathscr{A}$ onto $\mathscr{A}_{s]}, s \in T$ satisfying $E 1$ to $E 3$ (and $E 4$ in the stationary case) and the Markov property $M^{\prime}$. For a $W^{*}$-process, the $\left\{E_{s]}: s \in T\right\}$ are required to be normal.

Theorem 2.1 holds for a Markov process with conditional expectations, apart from normality of the maps $Z_{s, t}$, which is only ensured for a $W^{*}$-process. Moreover, if $T=\mathbb{R}$ and the process is also stationary, the $Z_{s, s+t}$ is independent of $s$ for all $t \geqq 0$ by $E 4$, so that, defining 


$$
Z_{t}=Z_{s, s+t} \quad \text { for all } s \text { in } \mathbb{R}, \quad t \geqq 0,
$$

$\left\{Z_{t}: t \geqq 0\right\}$ is a semigroup of completely positive identity preserving maps of $\mathscr{B}$ into itself, leaving invariant the state $\omega_{0}=\omega \circ j_{0}\left(=\omega \circ j_{t}\right.$ for all $t$ in $\mathbb{R}$ ).

The following is a straightforward consequence of Theorem 2.1.

Corollary 2.2.1. The time-ordered correlation kernels $w_{\mathbf{t}}, \mathbb{t} \in \mathbb{T}, \mathbb{t}=$ $\left(t_{1}, \ldots, t_{n}\right), t_{1} \leqq \cdots \leqq t_{n}$, of a Markov process with conditional expectations are given $b y$

$$
w_{\mathbf{t}}(\mathfrak{a} ; \mathfrak{b})=\omega \circ j_{t_{1}}\left(a_{1}^{*} Z_{t_{1}, t_{2}}\left(a_{2}^{*} \cdots Z_{t_{n-1}, t_{n}}\left(a_{n}^{*} b_{n}\right) \cdots b_{2}\right) b_{1}\right)
$$

for all $\mathrm{a}=\left(a_{1}, \ldots, a_{n}\right), \mathrm{b}=\left(b_{1}, \ldots, b_{n}\right)$ in $\mathscr{B}_{\mathfrak{a}} ;$ in particular, if the process is also stationary,

$$
w_{\mathrm{t}}(\mathrm{a} ; \mathrm{b})=\omega_{0}\left(a_{1}^{*} Z_{t_{2}-t_{1}}\left(a_{2}^{*} \cdots Z_{t_{n}-t_{n-1}}\left(a_{n}^{*} b_{n}\right) \cdots b_{2}\right) b_{1}\right) .
$$

The relations (2.8), (2.9) are known in the literature of physics $[25,26,27]$ as the "quantum regression theorem".

Definition. A stochastic process $\left(\mathscr{A},\left\{j_{t}\right\}, \omega\right)$ over $\mathscr{B}$ indexed by $T$ is said to satisfy the regression relation if there is a family $\left\{Z_{s, t}: s<t\right\}$ of maps of $\mathscr{B}$ into itself such that equation (2.8) holds.

Definition. We say that $\mathscr{A}$ is generated as a vector space by the timeordered products if, for each $s$ in $T, \mathscr{A}_{s]}$ (resp. $\mathscr{A}_{[s}$ ) is the closure, in the appropriate topology, of the linear span of the products $j_{t_{n}}\left(b_{n}\right) \cdots j_{t_{1}}\left(b_{1}\right)$, with $b_{1}, \ldots, b_{n}$ in $\mathscr{B}$ and $t_{1} \leqq \cdots \leqq t_{n} \leqq s$ (resp. $s \leqq t_{1} \leqq \cdots \leqq t_{n}$ ).

Theorem 2.2.2. Let $\left(\mathscr{A},\left\{j_{t}\right\}, \omega\right)$ be a stochastic process such that for each $s$ in $T$ the GNS representation of $\mathscr{A}_{s]}$ determined by $\omega \uparrow \mathscr{A}_{s]}$ is faithful, and $\mathscr{A}$ is generated as a vector space by the time-ordered products. Then, if the regression relation holds, $\left(\mathscr{A},\left\{j_{t}\right\}, \omega\right)$ is a Markov process with conditional expectations.

Proof. We give the details of the argument for a $W^{*}$-stochastic process; the proof for the $C^{*}$-case is an obvious modification. With the usual identification of $\mathscr{A}$ with $\pi(\mathscr{A})$, acting on $\mathscr{H}$ with cyclic vector $\Omega$, let $\mathscr{H}_{s]}$ be the closed subspace $\overline{\mathscr{A}_{s]} \Omega}$ of $\mathscr{H}$, and $P_{s]}$ the orthogonal projection of $\mathscr{H}$ onto $\mathscr{H}_{s]}$, for each $s$ in $T$. For $x$ in $\mathscr{B}(\mathscr{H})$, regard $P_{s]} x P_{s]}$ as an element of $\mathscr{B}\left(\mathscr{H}_{s]}\right)$, and define $\pi_{s]}: \mathscr{A}_{s]} \rightarrow \mathscr{B}\left(\mathscr{H}_{s]}\right)$ by $\pi_{s]}(a)=P_{s]} a P_{s]}$ for $a$ in $\mathscr{A}_{s]}$ : then $\left(\mathscr{H}_{s]}, \pi_{s]}, \Omega\right)$ is the GNS triple associated with $\left(\mathscr{A}_{s]}, \omega\left\lceil\mathscr{A}_{s]}\right)\right.$, since $P_{s]}$ commutes with all ele- 
ments of $\mathscr{A}_{s]}$ and leaves $\Omega$ invariant. The map $\pi_{s]}$ is normal, and faithful by assumption, hence $\pi_{s]}^{-1}$ exists and is a normal *-isomorphism. It follows from (2.8) and the assumption of the theorem that

$$
\begin{aligned}
& P_{s]} j_{t_{1}}\left(a_{1}\right)^{*} \cdots j_{t_{n}}\left(a_{n}\right)^{*} j_{t_{n}}\left(b_{n}\right) \cdots j_{t_{1}}\left(b_{1}\right) P_{s]} \\
& \quad=P_{s]}\left(j_{s} \circ Z_{s, t_{1}}\right)\left(a_{1}^{*} Z_{t_{1}, t_{2}}\left(a_{2}^{*} \cdots Z_{t_{n-1}, t_{n}}\left(a_{n}^{*} b_{n}\right) \cdots\right) b_{1}\right) P_{s]}
\end{aligned}
$$

for all $n$-tuples $\left(t_{1}, \ldots, t_{n}\right)$ in $\mathbf{T}$ such that $s \leqq t_{1} \leqq \cdots \leqq t_{n}$, and all $a_{1}, \ldots, a_{n}, b_{1}, \ldots, b_{n}$ in $\mathscr{B}$.

We conclude from this and the assumption of the theorem that

$$
P_{s]} \mathscr{A}_{[s} P_{s]} \subseteq \pi_{s]}\left(\mathscr{A}_{s}\right) \subseteq \pi_{s]}\left(\mathscr{A}_{s]}\right)
$$

since the maps $a \mapsto P_{s]} a P_{s]}$ and $a \mapsto \pi_{t]}(a)$ are bounded and normal, $\pi_{s]}$ is a representation and $\mathscr{A}_{s}$ is a $W^{*}$-subalgebra of the $W^{*}$-algebra $\mathscr{A}_{s]}$. For all $a$ in $\mathscr{A}_{[s}$ and $b$ in $\mathscr{A}_{s]}$, we find that $P_{s]} a b P_{s]}$ lies in $\pi_{s]}\left(\mathscr{A}_{s]}\right)$ since

$$
P_{s]} a b P_{s]}=P_{s]} a P_{s]} b P_{s]}=P_{s]} a P_{s]} \pi_{s]}(b) .
$$

By assumption, $\mathscr{A}$ is the closure of the linear span of $\left\{a b: a \in \mathscr{A}_{[s}, b \in \mathscr{A}_{s]}\right\}$, hence $P_{s]} \mathscr{A} P_{s]}=\pi_{s]}\left(\mathscr{A}_{s]}\right)$. Now define $E_{s]}: \mathscr{A} \rightarrow \mathscr{A}_{s]}$ by

$$
E_{s]}(a)=\pi_{s]}^{-1}\left(P_{s]} a P_{s]}\right) \quad \text { for all } a \text { in } \mathscr{A} \text {; }
$$

it is completely positive, identity preserving and normal, and by $(2.10)$ it satisfies the Markov property $M^{\prime}$. For $a$ in $\mathscr{A}$ and $b$ in $\mathscr{A}_{s}$, we have

$$
E_{s]}(a b)=\pi_{s]}^{-1}\left(P_{s]} a P_{s]} b P_{s]}\right)=\pi_{s]}^{-1}\left(P_{s]} a P_{s]}\right) b=E_{s]}(a) b,
$$

so that $E_{s]}$ satisfies $E 1$ (that is, it is a conditional expectation of $\mathscr{A}$ onto $\mathscr{A}_{s]}$ ); it satisfies the compatibility condition $E 2$ since $P_{s]} \Omega=\Omega$. The projectivity condition $E 3$ follows from the fact that the $\left\{E_{s]}: s \in T\right\}$ are uniquely determined. The same argument works for the $C^{*}$-case, with the appropriate change of topology.

2.3. In this and in the following sub-section we study a perturbation theory for stationary Markov processes with conditional expectations, which is based on a generalization $[8,9]$ of the ideas underlying the Feynman-Kac formula.

Definition. Let $\left(\mathscr{A},\left\{j_{t}\right\}, \omega\right)$ be a stationary Markov process indexed by $\boldsymbol{R}$, with group of automorphisms $\left\{u_{t}: t \in \boldsymbol{R}\right\}$ and conditional expectations $\left\{E_{t]}: t \in \boldsymbol{R}\right\}$. A family $\left\{m_{t}: t \geqq 0\right\}$ of completely positive identity preserving maps of $\mathscr{A}$ into itself is said to be a Markovian cocycle relative to $\left(\mathscr{A},\left\{j_{t}\right\}, \omega\right)$ if the following conditions are satisfied 
MC1: $\quad m_{s+t}=m_{s} u_{s} m_{t} u_{-s} \quad$ for all $s, t \geqq 0$;

MC2: $m_{t} \quad$ maps $\mathscr{A}_{[0}$ into itself for all $t \geqq 0$;

MC3: $m_{t}$ commutes with $E_{t\rfloor}$ for each $t \geqq 0$.

Theorem 2.3. Let $\left\{m_{t}: t \geqq 0\right\}$ be a Markovian cocycle relative to a stationary Markov process with conditional expectations $\left(\mathscr{A},\left\{j_{t}\right\}, \omega\right)$ over $\mathscr{B}$. Then

$$
\tilde{Z}_{t}=j_{0}^{*} E_{01} m_{t} j_{t}, t \geqq 0,
$$

defines a semigroup of completely positive identity preserving maps of $\mathscr{B}$ into itself, and

$$
\begin{array}{r}
E_{0]}\left(m_{t_{1}} j_{t_{1}}\left(a_{1}\right)^{*} \cdots m_{t_{n}} j_{t_{n}}\left(a_{n}\right)^{*} m_{t_{n}} j_{t_{n}}\left(b_{n}\right) \cdots m_{t_{1}} j_{t_{1}}\left(b_{1}\right)\right) \\
=j_{0} \tilde{Z}_{t_{1}}\left(a_{1}^{*} \tilde{Z}_{t_{2}-t_{1}}\left(a_{2}^{*} \cdots \tilde{Z}_{t_{n}-t_{n-1}}\left(a_{n}^{*} b_{n}\right) \cdots b_{2}\right) b_{1}\right)
\end{array}
$$

for all $0 \leqq t_{1} \leqq \cdots \leqq t_{n}$ in $\boldsymbol{R}, a_{1}, \ldots, a_{n}, b_{1}, \ldots, b_{n}$ in $\mathscr{B}$, and for all $n$.

Proof $([8,9])$. For all $b$ in $\mathscr{B}, m_{t} j_{t}(b)$ is in $\mathscr{A}_{[0}$, by $M C 2$, so that $E_{0]} m_{t} j_{t}(b)$ is in $\mathscr{A}_{0}$ by the Markov property $M^{\prime}$, and $\tilde{Z}_{t}$ is well-defined; it is completely positive and identity preserving by construction. Moreover, for all $s, t \geqq 0$, we have

$$
\begin{aligned}
\tilde{Z}_{s} \tilde{Z}_{t} & =j_{0}^{*} E_{0]} m_{s} u_{s} j_{0} j_{0}^{*} E_{0]} m_{t} u_{t} j_{0} & & \\
& =j_{0}^{*} E_{0]} m_{s} u_{s} E_{0 j} m_{t} u_{t} j_{0} & & \left(\text { since } j_{0} j_{0}^{*} \text { is the identity map of } \mathscr{A}_{0}\right) \\
& =j_{0}^{*} E_{0]} m_{s} E_{s]} u_{s} m_{t} u_{t} j_{0} & & (\text { by } E 4) \\
& =j_{0}^{*} E_{0]} E_{s]} m_{s} u_{s} m_{t} u_{t} j_{0} & & (\text { by } M C 3) \\
& =j_{0}^{*} E_{0]} m_{s} u_{s} m_{t} u_{t} j_{0} & & (\text { by } E 3) \\
& =j_{0}^{*} E_{0]} m_{s+t} u_{s+t} j_{0} & & (\text { by } M C 1) \\
& =\tilde{Z}_{s+t} & &
\end{aligned}
$$

Equation (2.13) is obtained by replacing $E_{0]}$ in the left-hand side by $E_{0]} E_{t_{1}} \cdots$ $E_{\left.t_{n-1}\right]}$, which is permissible by $E 3$, and by using $E 1, M C 3$ and (2.12) (cf. the proof of Theorem 2.1).

2.4. Consider a stationary Markov process whose group of automorphisms $\left\{u_{t}: t \in \boldsymbol{R}\right\}$ is strongly continuous with infinitesimal generator $\delta$. Then also $\left\{Z_{t}=j_{0}^{*} E_{0]} u_{t} j_{0}: t \geqq 0\right\}$ is strongly continuous, with infinitesimal generator $L$. Let $v$ be a self-adjoint element of $\mathscr{B}$, and let $\left\{\tilde{u}_{t}: t \in \mathbb{R}\right\}$ be the strongly continuous group of *automorphisms of $\mathscr{A}$ whose infinitesimal generator is

$$
\tilde{\delta}=\delta+i\left[j_{0}(v), \cdot\right] \quad \text { on } \mathscr{D}(\delta) .
$$


Lemma 2.4.1. The family $\left\{m_{t}: t \geqq 0\right\}$ defined by

$$
m_{t}=\tilde{u}_{t} u_{-t}, t \geqq 0,
$$

is a Markovian cocycle. The corresponding semigroup $\left\{\tilde{Z}_{t}=j_{0}^{*} E_{0]} m_{t} j_{t}: t \geqq 0\right\}$ is strongly continuous, with infinitesimal generator

$$
\tilde{L}=L+i[v, \cdot] \quad \text { on } \mathscr{D}(L) .
$$

Proof. For all $a$ in $\mathscr{A}$ and $t \geqq 0$, we have (see e.g. [28]),

$$
\begin{aligned}
m_{t}(a) & =a+\sum_{n=1}^{\infty} i^{n} \int_{0 \leqq t_{1} \leqq \cdots \leqq t_{n} \leqq t} \cdots \int_{t_{1}}\left[j_{t_{1}}(v),\left[\cdots,\left[j_{t_{n}}(v), a\right] \cdots\right]\right] d t_{1} \cdots d t_{n} \\
& =M_{t} a M_{t}^{*}
\end{aligned}
$$

where

$$
M_{t}=1+\sum_{n=1}^{\infty} i^{n} \int_{0 \leqq t_{1} \leqq \cdots \leqq t_{n} \leqq t} \ldots \int_{t_{1}} j_{1}(v) \cdots j_{t_{n}}(v) d t_{1} \cdots d t_{n} \in \mathscr{A}_{[0} \cap \mathscr{A}_{t]} .
$$

Then it is clear that $\left\{m_{t}: t \geqq 0\right\}$ satisfies $M C 2$ and $M C 3$, and it satisfies $M C 1$ since both $\left\{u_{t}\right\}$ and $\left\{\tilde{u}_{t}\right\}$ are groups. Finally, for any $b$ in $\mathscr{B}$ we have

$$
\begin{aligned}
t^{-1}\left[\tilde{Z}_{t}(b)-b\right] & =t^{-1}\left[Z_{t}(b)-b\right]+t^{-1}\left[\tilde{Z}_{t}(b)-Z_{t}(b)\right] \\
& =t^{-1}\left[Z_{t}(b)-b\right]+j_{0}^{*} E_{0]}\left[t^{-1}\left(m_{t}-1\right) j_{t}(b)\right] .
\end{aligned}
$$

It follows from the explicit expression (2.16) of $m_{t}$ that the second term of the right-hand side tends to the limit $i[v, b]$ as $t \rightarrow 0$, then (2.15) follows.

Lemma 2.4.2. Define $\tilde{j}_{t}=\tilde{u}_{t} j_{0}, t \in \mathbb{R}$, and let

$$
\tilde{\mathscr{A}}_{t}=\tilde{j}_{t} \mathscr{B}, \tilde{\mathscr{A}_{t]}}=\vee\left\{\tilde{\mathscr{A}}_{s}: s \leqq t\right\}, \tilde{\mathscr{A}}_{[t}=\vee\left\{\tilde{\mathscr{A}}_{u}: t \leqq u\right\}
$$

Then

$$
\tilde{\mathscr{A}}_{t]}=\mathscr{A}_{t]} \text { for } t \geqq 0 \text { and } \tilde{\mathscr{A}}_{[t}=\mathscr{A}_{[t} \text { for } t \leqq 0 .
$$

Proof. From the proof of Lemma 2.4.1 it is clear that $\tilde{\mathscr{A}}_{t]} \subseteq \mathscr{A}_{t]}$ for all $t \geqq 0$. By interchanging the roles of $\tilde{u}_{t}$ and $u_{t}$, the converse inclusion follows. The second half of (2.19) is shown in the same way, considering the perturbative series for $\tilde{u}_{t} u_{-t}$ and $u_{t} \tilde{u}_{-t}$ for negative times (cf. [28]).

Lemma 2.4.3. There exists at least one state $\tilde{\omega}$ on $\mathscr{A}$ which is invariant under $\tilde{u}_{t}$ and compatible with $E_{0 \mathrm{~J}}$. Then also $\tilde{Z}_{t}$ has at least one stationary state, namely $\tilde{\omega}_{\circ} \tilde{j}_{0}$.

Proof. Let $\tilde{\omega}$ be a weak* limit point as $t \rightarrow \infty$ of the net $\left\{\frac{1}{t} \int_{0}^{t} \omega \circ \tilde{u}_{s} d s\right.$ : 
$t \geqq 0\}$, the integrals being weak* Riemann integrals. Such a limit point always exists by the weak* compactness of the set of states on a $C^{*}$-algebra with identity. Then $\tilde{\omega}$ is invariant under $u_{t}$ by construction. Since, for all $s \geqq 0$,

$$
\tilde{u}_{s} E_{0]}=m_{s} u_{s} E_{0]}=m_{s} E_{s]} u_{s}=E_{s]} m_{s} u_{s}=E_{s]} \tilde{u}_{s}
$$

and $\omega$ is compatible with all $E_{s]}, \tilde{\omega}$ is also compatible with $E_{0]}$. Finally, $\tilde{\omega} \circ j_{0} \circ \tilde{Z}_{t}=\tilde{\omega} \circ E_{0]} \tilde{u}_{t} j_{0}=\tilde{\omega} \circ j_{0}$.

Theorem 2.4.4. Under the assumptions and with the notations introduced above, $\left(\mathscr{A},\left\{\tilde{J}_{t}\right\}, \tilde{\omega}\right)$ is a new stationary Markov process over $\mathscr{B}$, with associated group of automorphisms $\left\{\tilde{u}_{t}: t \in \mathbb{R}\right\}$ of $\mathscr{A}$, semigroup $\left\{\tilde{Z}_{t}: t \geqq 0\right\}$ of $\mathscr{B}$, and conditional expectations $\left\{\widetilde{E}_{t]}: \mathscr{A} \rightarrow \tilde{\mathscr{A}_{t}} ; t \in \mathbb{R}\right\}$ which coincide with $E_{t]}$ for $t \geqq 0$.

Proof. By Lemma 2.3, $\left(\mathscr{A},\left\{\tilde{J}_{t}\right\}, \tilde{\omega}\right)$ is a stationary process with group of automorphisms $\left\{\tilde{u}_{t}\right\}$, and $\tilde{\omega}$ is compatible with the maps

$$
\widetilde{E}_{t]}=\tilde{u}_{t} E_{0]} u_{-t}, t \in \mathbb{R},
$$

which are conditional expectations onto $\tilde{\mathscr{A}_{t]}}=\tilde{u}_{t} \mathscr{A}_{0]}, t \in \mathbb{R}$, and satisfy the covariance condition $E 4$ with respect to $\left\{\tilde{u}_{t}\right\}$. Moreover, we have

$$
\tilde{E}_{t]} \tilde{\mathscr{A}}_{[t}=\tilde{u}_{t} E_{0]} \tilde{\mathscr{A}}_{[0}=\tilde{u}_{t} E_{0]} \mathscr{A}_{[0}=\tilde{u}_{t} \mathscr{A}_{0}=\tilde{\mathscr{A}}_{t}
$$

by Lemma 2.2 and the Markov property of the original process. Similarly, for $s \leqq t$ in $\mathbb{R}$, we have, by (2.20),

$$
\widetilde{E}_{s]} \widetilde{E}_{t]}=\tilde{u}_{s} E_{0]} \tilde{u}_{t-s} E_{0]} \tilde{u}_{-t}=\tilde{u}_{s} E_{0]} E_{s]} \tilde{u}_{-s}=\tilde{u}_{s} E_{0]} \tilde{u}_{-s}=\widetilde{E}_{s]},
$$

so that the projectivity condition $E 3$ holds. Then $\left(\mathscr{A},\left\{\tilde{j}_{t}\right\}, \tilde{\omega}\right)$ is a stationary Markov process with conditional expectations. The semigroup associated to it is $\left\{\tilde{Z}_{t}\right\}$ given by (2.12), since $\widetilde{E}_{0]}=E_{0]}$. We have also, by (2.20), $\widetilde{E}_{t]}=$ $\tilde{u}_{t} E_{0]} \tilde{u}_{-t}=E_{t]} \tilde{u}_{-t} \tilde{u}_{t}=E_{t]}$ for all $t \geqq 0$.

\section{Comments and Remarks}

To 2.1: In the classical case $\left(\mathscr{A}, \mathscr{B}\right.$ abelian $W^{*}$-algebras, $\omega$ a faithful normal state) the canonical maps $E_{s, t}$ are always conditional expectations, and our definition of the Markov property is equivalent to the usual one for processes in the sense of Doob, indexed by a totally ordered set. However, it should be mentioned that the Markov property is of interest also for stochastic processes indexed by a set $T$ which is not totally ordered (multi-dimensional Markov 
property); in particular, it is relevant for those classical generalized stochastic processes which are the Euclidean version of quantum field theories (see e.g. $[29,30])$. For a non-commutative formulation of the multi-dimensional Markov property, see [23].

To 2.2: By Corollary 2.2.1 and Theorem 2.2.2, the regression relation (2.8), or (2.9) in the stationary case, is characteristic of Markov processes with conditional expectations, under some conditions on the commutation relation in $\mathscr{A}$. In particular, (2.8) holds for all classical Markov processes, ensuring that a classical Markov process is uniquely determined by its associated evolution $\left\{Z_{s, t}: s \leqq t \in T\right\}$ on $\mathscr{B}$ and initial distribution $\omega_{0}=\omega \circ j_{0}(0 \equiv \min T)$, or stationary distribution in the stationary case. This is no more the case for non-commutative Markov processes; see for instance [3, 20] for the structure of symmetric quantum Markov processes and the formula replacing (2.8) in that situation. However, non-commutative stochastic processes satisfying the regression relation have an obvious interest. Lindblad [31] takes the regression relation as a definition of the Markov property, in the framework of a theory of stochastic processes whose basic objects are the time-ordered correlation kernels.

It is worth mentioning the fact that the regression relation (2.9) is a strictly stronger propercy than the semigroup property for the reduced dynamics:

$S G:$ There exists a semigroup $\left\{Z_{t}: t \geqq 0\right\}$ and an invariant state $\omega_{0}$ on $\mathscr{B}$ such that

$$
w_{t_{1}, t_{2}}\left(a_{1}, a_{2} ; b_{1}, b_{2}\right)=\omega_{0}\left(a_{1}^{*} Z_{t_{2}-t_{1}}\left(a_{2}^{*} b_{2}\right) b_{1}\right)
$$

for all $a_{1}, a_{2}, b_{1}, b_{2}$ in $\mathscr{B}$ and $t_{1} \leqq t_{2}$ in $\boldsymbol{R}$.

This was already known for classical stochastic processes [32]; Lindblad [33] has provided a quantum-mechanical example of a process satisfying SG, and not the regression relation, which retains a perfect memory of the initial state; it is non-Markovian also with our definition of a Markov process. A version of Lindblad's example is given in the Appendix.

It is an interesting question whether it is possible to construct a stationary Markov process (with conditional expectations) over $\mathscr{B}$, indexed by $\boldsymbol{R}$, given a semigroup $\left\{Z_{t}: t \geqq 0\right\}$ of completely positive identity preserving maps of $\mathscr{B}$, with an invariant state $\omega_{0}$, in such a way that the regression relation (2.9) holds. This is known to be the case for classical stochastic processes (KolmogorovDaniell construction): the semigroup and the stationary state give the timeordered correlation kernels, and all the correlation kernels are obtained by 
permutation symmetry. More generally, Theorem 2.2.2 suggests that a construction is possible if some commutation relations in $\mathscr{A}$ are given in advance, so that all the correlation kernels can be constructed from the time-ordered ones, and $\mathscr{A}$ is generated as a vector space by the time-ordered products of $j_{t}(b)$, $b \in \mathscr{B}, t \in \mathbb{R}$. In the following section we perform this construction for a class of processes on the Clifford algebra, which we call quasi-free. A similar construction for non-commutative Gaussian processes on the CCR algebra can be found in [34].

To 2.3: For other work on quantum versions of the Feynman-Kac formula, see $[35,36,20,31,38]$. We refer to [8] for a detailed discussion of the structure presented in Theorem 2.3.

Markovian cocycles consisting of maps which do not preserve the identity could also be considered $[8,9]$, then the semigroup $\tilde{Z}_{t}$ of Theorem 2.3 would not be identity preserving.

To 2.4: Lemmas 2.4.1 and 2.4.2 have an obvious $W^{*}$-version under the condition that $\left\{u_{t}: t \in \boldsymbol{R}\right\}$ is continuous in the $W^{*}$-topology. Then also unbounded self-adjoint $v$ 's affiliated to $\mathscr{A}_{0}$ could be considered. The convergence of the right-hand side of (2.16) is all that is needed in order to define the Markovian cocycle $m_{t}$, and hence the weakly* continuous semigroup $\tilde{Z}_{t}$; then the generator $\tilde{L}$ of $\tilde{Z}_{t}$ can be taken as a definition of the sum of $L$ and $i[v, \cdot]$ (cf. [39] in the classical case).

Lemma 2.4 .3 is proved using the $W^{*}$-compactness of the set of states on a $C^{*}$-algebra and the strong continuity of $\left\{\tilde{u}_{t}: t \in \mathbb{R}\right\}$ hence it has no obvious $W^{*}$ version; thus also Theorem 2.4 .4 has no obvious $W^{*}$-version.

An analoguc of Lemma 2.4.1 still holds if one allows an explicit time dependence of $v \in \mathscr{B}$. If $t \mapsto v(t)=v(t)^{*}$ is a continuous function on $\mathbb{R}$, with values in $\mathscr{B}$, we can put

$$
\begin{array}{r}
m_{s, t}(a)=a+\sum_{n=1}^{\infty} i^{n} \int_{s \leqq t_{1} \leqq \cdots \leqq t_{n} \leqq t} \cdots \int_{t_{1}}\left[j _ { t _ { 1 } } \left(v\left(t_{1}\right),\left[\cdots,\left[j_{t_{n}}\left(v\left(t_{n}\right), a\right] \cdots\right]\right] d t_{1} \cdots d t_{n}\right.\right. \\
\text { for } s \leqq t \text { in } \mathbb{R}, \\
m_{s, t}(a)=a+\sum_{n=1}^{\infty} i^{n} \int_{s \geqq t_{1} \geqq \cdots \geqq t_{n} \geqq t} \cdots \int_{t_{1}}\left[j _ { t _ { 1 } } \left(v\left(t_{1}\right),\left[\cdots,\left[j_{t_{n}}\left(v\left(t_{n}\right), a\right] \cdots\right]\right] d t_{1} \cdots d t_{n}\right.\right. \\
\text { for } s \geqq t \text { in } \mathbb{R} .
\end{array}
$$

Then $m_{s, t}$ is completely positive, identity preserving, and

$$
m_{s, t} m_{t, u}=m_{s, u} \quad \text { for } \quad s \leqq t \leqq u \quad \text { in } \quad \mathbb{R} ;
$$


$m_{s, t}$ maps $\mathscr{A}_{[s}$ into itself and commutes with $E_{t]}$ for each $s \leqq t$ in $\mathbb{R}$. Proceeding in analogy with Theorem 2.3, Lemma 2.4.1, we can put

$$
Z_{s, t}=j_{s}^{*} E_{s]} m_{s, t} j_{t}, \quad s \leqq t \quad \text { in } \mathbb{R} ;
$$

then

$$
\frac{d}{d t} \tilde{Z}_{s, t}(b)=\tilde{Z}_{s, t}(L(b)+i[v(t), b]) \quad \text { for all } b \text { in } \mathscr{D}(L), \quad s \leqq t \text { in } \boldsymbol{R},
$$

and

$$
\tilde{Z}_{s, t} \tilde{Z}_{t, u}=\tilde{Z}_{s, u} \quad \text { for all } s \leqq t \leqq u \text { in } \mathbb{R} .
$$

Letting $\tilde{j}_{t}=m_{0, t} j_{t}=m_{0, t} u_{t} j_{0}$, we find also in analogy to (2.13)

$$
\begin{aligned}
& E_{0]}\left(\tilde{j}_{t_{1}}\left(a_{1}\right)^{*} \ldots \tilde{j}_{t_{n}}\left(a_{n}\right)^{*} \tilde{j}_{t_{n}}\left(b_{n}\right) \cdots \tilde{j}_{t_{1}}\left(b_{1}\right)\right) \\
& \quad=\tilde{j}_{0} \circ \tilde{Z}_{0, t_{1}}\left(a_{1}^{*} \tilde{Z}_{t_{1}, t_{2}}\left(a_{2}^{*} \cdots \tilde{Z}_{t_{n-1}, t_{n}}\left(a_{n}^{*} b_{n}\right) \cdots b_{2}\right) b_{1}\right)
\end{aligned}
$$

for all $0 \leqq t_{1} \leqq \cdots \leqq t_{n}$ in $\boldsymbol{R}, a_{1}, \ldots, a_{n}, b_{1}, \ldots, b_{n}$ in $\mathscr{B}$, and for all $n$. The process $\left(\mathscr{A},\left\{\tilde{J}_{t}\right\}, \omega\right)$ is neither stationary nor Markov, in general, but conditional expectations onto $\tilde{\mathscr{A}}_{t]}$ which are compatible with $\omega$ exist for $t \geqq 0$, since $\tilde{\mathscr{A}}_{t]}=$ $\mathscr{A}_{t]}$ for $t \geqq 0$. Since $\left\{\tilde{u}_{t}=m_{0, t} u_{t}: t \in \mathbb{R}\right\}$ is not a group of automorphisms of $\mathscr{A}$, there will be no choice of a state $\tilde{\omega}$ on $\mathscr{A}$ which makes $\left(\mathscr{A},\left\{\tilde{J}_{t}\right\}, \tilde{\omega}\right)$ a stationary process, moreover, a limit point of $\left\{\frac{1}{t} \int_{0}^{t} \omega \circ \tilde{u}_{s} d s\right\}$ need not be invariant under $\left\{\tilde{u}_{t}: t \in \boldsymbol{R}\right\}$. So there is no analogue of Theorem 2.4 .4 for time-dependent perturbations.

\section{§3. Processes on the Clifford Algebra}

3.1. We recall some preliminary information about the Clifford algebra and the quasi-free states and quasi-free maps on it $[40, \ldots, 45]$.

The Clifford algebra $A(H)$ over a real Hilbert space $H$ is the (unique up to *-isomorphism) $C^{*}$-algebra generated by the identity 1 and the self-adjoint elements $B(h)$, linear in $h \in H$, satisfying the canonical anticommutation relations

$$
B(h) B(k)+B(k) B(h)=2(h, k) 1 \quad \text { for all } h, k \text { in } H \text {. }
$$

The map $h \mapsto B(h)$ is continuous and $\|B(h)\|=\|h\|$ for all $h$ in $H$.

Let $M, H$ be real Hilbert spaces. For any isometry $X$ of $M$ into $H$ there is a unique *-isomorphism, denoted by $A(X)$, of $A(M)$ into $A(H)$, such that

$$
A(X)\left(B\left(m_{1}\right) \cdots B\left(m_{n}\right)\right)=B\left(X m_{1}\right) \cdots B\left(X m_{n}\right) \quad \text { for all } m_{1}, \ldots, m_{n} \text { in } M .
$$


A state $\omega$ on $A(H)$ is said to be quasi-free if

(3.3a) $\omega\left(B\left(h_{1}\right) \cdots B\left(h_{2 n+1}\right)\right)=0$ for all $n=0,1, \ldots$ and all $h_{1}, \ldots, h_{2 n+1}$ in $H$,

(3.3b) $\quad \omega\left(B\left(h_{1}\right) \cdots B\left(h_{2 n}\right)\right)=\sum_{p \in \mathscr{P}_{n}}(\operatorname{sgn} p) \prod_{r=1}^{n} \omega\left(B\left(h_{p(2 r-1)}\right) B\left(h_{p(2 r)}\right)\right)$ for all $n=1,2, \ldots$ and all $h_{1}, \ldots, h_{2 n}$ in $H$;

where $\mathscr{P}_{n}$ is the set of those permutations $p$ of $\{1, \ldots, 2 n\}$ such that $p(2 r-1)<$ $p(2 r)$ and $p(2 r-1)<p(2 r+1)$ for all $r=1, \ldots, n$, and $\operatorname{sgn} p$ is the parity of $p$.

There is a one-to-one correspondence between quasi-free states $\omega$ on $A(H)$ and skew-adjoint contractions $Q$ on $H$, given by

$$
\omega(B(h) B(k)) \equiv \omega_{Q}(B(h) B(k))=(h, k)+i(Q h, k) .
$$

Notice that a skew-symmetric bilinear form $q(\cdot, \cdot)$ on $H$ is given by $q(h, k)=$ $(Q h, k), Q$ being a skew-adjoint contraction on $H$, if and only if

$$
\sum_{i, j} \bar{c}_{i} c_{j}\left[\left(h_{i}, h_{j}\right)+i q\left(h_{i}, h_{j}\right)\right] \geqq 0,
$$

for all finite sequences $\left\{c_{i} \in \mathbb{C}: i=1, \ldots, n\right\}$ and $\left\{h_{i} \in H: i=1, \ldots, n\right\}$.

The GNS representation of $A(H)$ determined by a quasi-free state $\omega_{Q}$ is faithful, since $\omega_{Q}$ is a product state of a faithful state and a state on a simple $C^{*}$-algebra [42].

The following Lemma is a rephrasing of results due to Evans [43, 44], Fannes and Rocca [45].

Lemma 3.1. If $Z$ is a completely positive identity preserving map of $A(H)$ into itself satisfying

$$
Z(B(h))=B(T h) \quad \text { for all } h \text { in } H,
$$

where $T$ is a linear operator on $H$ and

$$
\omega_{Q^{\circ} Z}=\omega_{Q}
$$

where $\omega_{Q}$ is a quasi-free state on $A(H)$, then

$$
\sum_{i, j} \bar{c}_{i} c_{j}\left[\left(h_{i}, h_{j}\right)-\left(T h_{i}, T h_{j}\right)+i\left(Q h_{i}, h_{j}\right)-i\left(Q T h_{i}, T h_{j}\right)\right] \geqq 0
$$

for all finite sequences $\left\{c_{i}\right\}$ in $\mathbb{C}$ and $\left\{h_{i}\right\}$ in $H$, which in particular implies that $T$ is a contraction. Conversely [43,44,45], given a contraction $T$ and $a$ skew-adjoint contraction $Q$ on $H$ satisfying (3.7), there is a canonical completely positive identity preserving map of $A(H)$ into itself, satisfying (3.6a), (3.6b). 
Proof. $Z$ maps the linear span of $\{B(h): h \in H\}$ into itself and is a contraction with respect to the semi-norm:

$$
\|x\|_{Q}^{2}=\omega_{Q}\left(x^{*} x\right), x \in \operatorname{lin}_{\boldsymbol{C}}\{B(h): h \in H\}
$$

since

$$
\omega_{Q}(Z(x) * Z(x)) \leqq \omega_{Q}\left(Z\left(x^{*} x\right)\right)=\omega_{Q}\left(x^{*} x\right)
$$

by the Kadison-Schwarz inequality. This fact is expressed by (3.7). Conversely, if (3.7) holds, then $T$ is a contraction and

$$
\omega_{Q}^{T}\left(B\left(h_{1}\right) \cdots B\left(h_{k}\right)\right)=\omega_{Q}\left(B\left(D h_{1}\right) \cdots B\left(D h_{k}\right)\right)
$$

where $D=\left(1-T^{*} T\right)^{1 / 2}$. Hence a completely positive identity preserving map $Z$ can be constructed by letting $[43,44,45]$

$$
Z\left(B\left(h_{1}\right) \cdots B\left(h_{n}\right)\right)=\sum_{\text {partitions }}(\operatorname{sgn} p) B\left(T h_{i_{1}}\right) \cdots B\left(T h_{i_{m}}\right) \omega_{Q}^{T}\left(B\left(h_{i_{m+1}}\right) \cdots B\left(h_{i_{n}}\right)\right),
$$
where $\omega_{Q}^{T}(\cdot)$ is defined by $(3.8 \mathrm{a})$, the summation extends to the partitions of $\{1, \ldots, n\}$ into two sets $\left\{i_{1}<\cdots<i_{m}\right\},\left\{i_{m+1}<\cdots<i_{n}\right\}$ and $\operatorname{sgn} p$ is the parity of the permutation $\{1, \ldots, n\} \mapsto\left\{i_{1}, \ldots, i_{n}\right\}$.

Definition. A map defined by equations (3.8a), (3.8b) is said to be a quasifree completely positive map, and denoted by $A_{Q}(T)$.

Notice that $A_{Q}(0)=\omega_{Q}(\cdot) 1$; if $T$ is an isometry commuting with $Q$ then $A_{Q}(T)$ coincides with $A(T)$ defined by (3.2), and $A_{Q}(T)$ is a conditional expectation, compatible with $\omega_{Q}$, if and only if $T$ is an orthogonal projection commuting with $Q$. The set $\mathscr{S}_{Q}$ of contractions $T$ on $H$ satisfying (3.7) is a semigroup, and $T \mapsto A_{Q}(T)$ is a homomorphism of $\mathscr{S}_{Q}$ into the set completely positive identity preserving maps on $A(H)$.

3.2. Let $\left\{X_{t}: t \in \boldsymbol{R}\right\}$ be a family of isometries from a real Hilbert space $M$ into a real Hilbert space $H$, such that $H=\vee\left\{X_{t} m: t \in \boldsymbol{R}, m \in M\right\}$ and let $Q$ be a skew-adjoint contraction on $H$. Let $\mathscr{A}=A(H)$, and let

$$
j_{t}=A\left(X_{t}\right): A(M) \longrightarrow A(H), \omega=\omega_{Q} .
$$

The stochastic process $\left(\mathscr{A},\left\{j_{t}\right\}, \omega\right)$ over $A(M)$, indexed by $\boldsymbol{R}$, is said to be a quasi-free process. Notice that $t \mapsto j_{t}$ is strongly continuous if and only if $t \mapsto X_{t}$ is.

Theorem 3.2.1. A quasi-free process over $A(M)$ is determined up to equivalence by its covariance function

$$
\omega\left(j_{t} B(m) j_{t^{\prime}} B\left(m^{\prime}\right)\right)=\left(m, K\left(t, t^{\prime}\right) m^{\prime}\right)+i\left(m, K^{Q}\left(t, t^{\prime}\right) m^{\prime}\right), m, m^{\prime} \in M, t, t^{\prime} \in \boldsymbol{R}
$$


where $K\left(t, t^{\prime}\right)$ and $K^{Q}\left(t, t^{\prime}\right)$ in $\mathscr{B}(M)$ satisfy

$$
K(t, t)=1 \text { for all } t \text { in } \mathbb{R} \text {, }
$$

$$
K\left(t, t^{\prime}\right)^{*}=K\left(t^{\prime}, t\right), K^{Q}\left(t, t^{\prime}\right)^{*}=-K^{Q}\left(t^{\prime}, t\right) \text { for all } t, t^{\prime} \text { in } \mathbb{R} \text {, }
$$

for all finite sequences $\left\{c_{i}\right\}$ in $\boldsymbol{C},\left\{t_{i}\right\}$ in $\mathbb{R}$ and $\left\{m_{i}\right\}$ in $M$.

Proof. If $\left(\mathscr{A},\left\{j_{t}\right\}, \omega\right)$ is a quasi-free process over $A(M)$, then $K\left(t, t^{\prime}\right)$ $=X_{t}^{*} X_{t^{\prime}}$ and $K^{Q}\left(t, t^{\prime}\right)=-X_{t}^{*} Q X_{t^{\prime}}$ satisfy all the above propertics. Conversely, given $K(\cdot, \cdot)$ and $K^{Q}(\cdot, \cdot)$ satisfying the above properties, $K(\cdot, \cdot)$ is a positive definite kernel on $\mathbb{R} \times \boldsymbol{R}$, and has a minimal Kolmogorov decomposition [13] $X_{t}: M \rightarrow H$, each $X_{t}$ being isometric. Then $K^{Q}$ defines a skew-symmetric form $q$ on $H$ via

$$
q\left(\sum_{i} c_{i} X_{t_{i}} m_{i}, \sum_{i} c_{j}^{\prime} X_{t_{j}^{\prime}} m_{j}^{\prime}\right)=\sum_{i, j} c_{i} c_{j}^{\prime}\left(m_{i}, K^{Q}\left(t_{i}, t_{j}^{\prime}\right) m_{j}^{\prime}\right),
$$

for all finite sequences $\left\{m_{i}\right\},\left\{m_{j}^{\prime}\right\}$ in $M,\left\{c_{i}\right\},\left\{c_{j}^{\prime}\right\},\left\{t_{i}\right\},\left\{t_{j}^{\prime}\right\}$ in $\mathbb{R}^{\mathrm{R}}$. By (3.11b), cf. (3.5), $q$ is given by a skew-adjoint contraction $Q$ on $H$. Then $H,\left\{X_{t}\right\}$ and $Q$ can be used to construct the quasi-free process; they determine all the correlation kernels, hence the quasi-free process is determined up to equivalence.

Theorem 3.2.2. A quasi-free process is stationary if and only if there exists a group $\left\{T_{t}: t \in \boldsymbol{R}\right\}$ of unitaries on $H$ such that

$$
\begin{array}{ll}
T_{t} X_{s}=X_{s+t} & \text { for all } t, s \text { in } \mathbb{R}, \\
T_{t} Q=Q T_{t} & \text { for all } t \text { in } \mathbb{R},
\end{array}
$$

and

$$
u_{t}=A\left(T_{t}\right) \quad \text { for all } t \text { in } \mathbb{R} \text {. }
$$

Proof. If the process is stationary, $K\left(s, s^{\prime}\right)=K\left(s+t, s^{\prime}+t\right)$ for all $s, s^{\prime}, t$ in $\boldsymbol{R}$. By the uniqueness of the minimal Kolmogorov decomposition of a positive definite kernel [13], there are unitaries $\left\{T_{t}: t \in \boldsymbol{R}\right\}$ on $H$ such that (3.12a) holds. The group property is checked in a straightforward way. Moreover, also $K^{Q}\left(s, s^{\prime}\right)=K^{Q}\left(s+t, s^{\prime}+t\right)$ for all $s, s^{\prime}, t$ in $\boldsymbol{R}$, hence $T_{t}^{-1} Q T_{t}=Q$ for all $t$ in $\boldsymbol{R}$, and (3.12b) holds. Then for all $s_{1}, \ldots, s_{n}, t$ in $\mathbb{R}, m_{1}, \ldots, m_{n}$ in $M$, we have

$$
\begin{aligned}
u_{t}\left(B\left(X_{s_{1}} m_{1}\right) \cdots B\left(X_{s_{n}} m_{n}\right)\right) & =u_{t} B\left(X_{s_{1}} m_{1}\right) \cdots u_{t} B\left(X_{s_{n}} m_{n}\right) \\
& =B\left(T_{t} X_{s_{1}} m_{1}\right) \cdots B\left(T_{t} X_{s_{n}} m_{n}\right)
\end{aligned}
$$

so that $(3.12 \mathrm{c})$ holds. Conversely, if $(3.12 \mathrm{a})$ and $(3.12 \mathrm{~b})$ hold, define $u_{t}$ by 
(3.12c) and check that $u_{t} j_{s}=j_{s+t}$ for all $s, t$ in $\boldsymbol{R}, \omega_{Q^{\circ} u_{t}}=\omega_{Q}$ for all $t$ in $\mathbb{R}$.

3.3. Now we study Markov processes with conditional expectations. Since $A=A(H)$ is generated as a vector space by the time-ordered products, such processes are exactly those for which the regression relation (2.8) holds, by Corollary 2.2.1 and Theorem 2.2.2. If $\omega_{Q}$ is a separating state (equivalently, if $\left.\operatorname{Ker}\left(1-Q^{*} Q\right)=\{0\}\right)$, it is possible to show that conditional expectations $E_{t]}$ onto $\mathscr{A}_{t]}=A\left(H_{t]}\right)$, compatible with $\omega_{Q}$, exist if and only if $P_{t]}$ commutes with $Q$, in which case $E_{t]}=A_{Q}\left(P_{t]}\right)$ for each $t$ in $\boldsymbol{R}$ (use Takesaki's theorem [22] and the explicit form of the modular automorphism group associated to a separating quasi-free state [46]). In the general case $\left(\operatorname{Ker}\left(1-Q^{*} Q\right)\right.$ arbitrary), for the sake of brevity we make the following

Definition. A quasi-free process $\left(\mathscr{A},\left\{j_{t}\right\}, \omega\right)$ is said to be a quasi-free Markov process if it is a Markov process with conditional expectations given by

$$
E_{t]}=A_{Q}\left(P_{t]}\right) \quad \text { for all } t \text { in } \mathbb{R} .
$$

Theorem 3.3.1. A quasi-free process is quasi-free Markov if and only if

$$
K(s, t) K(t, u)=K(s, u) \quad \text { for all } s \leqq t \leqq u \text { in } \mathbb{R}
$$

and

$$
K^{Q}(s, t)=-Q_{s} K(s, t) \quad \text { for all } s, t \text { in } \mathbb{R},
$$

$Q_{\text {s }}$ being a skew-adjoint contraction on $M$.

Proof. Suppose that the process is quasi-free Markov. Then $Q$ commutes with all $P_{t]}$, and $A_{Q}\left(P_{t]}\right) A\left(H_{[t}\right)=A\left(H_{t}\right)$ implies $P_{t]} H_{[t}=H_{t}$, where $H_{[t}=\vee\left\{X_{u} M\right.$ : $t \leqq u\}$ and $H_{t}=X_{t} M$. Then $H$ can be decomposed as $H=D_{t}^{-} \oplus H_{t} \oplus D_{t}^{+}$, where $D_{t}^{-}=H_{t]} \ominus H_{t}, D_{t}^{+}=H_{[t} \ominus H_{t}$. Hence $\left(\left(1-P_{t}\right) X_{s} m,\left(1-P_{t}\right) X_{u} m^{\prime}\right)=0$ for all $m, m^{\prime}$ in $M, s \leqq t \leqq u$ in $\boldsymbol{R}$, where $P_{t}=X_{t} X_{t}^{*}$ is the orthogonal projection of $H$ onto $H_{t}$. But this can be re-written as

$$
\left(m,(K(s, u)-K(s, t) K(t, u)) m^{\prime}\right)=0 \quad \text { for all } m, m^{\prime} \text { in } M,
$$

which is (3.14a). Moreover, for $s \leqq t$,

$$
K^{Q}(s, t)=-X_{s}^{*} Q X_{t}=-X_{s}^{*} P_{s]} Q X_{s}=-X_{s}^{*} Q P_{s]} X_{t}=-X_{s}^{*} Q X_{s} X_{s}^{*} X_{t},
$$

since $P_{s]} X_{t}=P_{s} X_{t}$ for $s \leqq t$ by the Markov property, which proves (3.14b), with $Q_{s}=X_{s}^{*} Q X_{s}$.

The argument can also be reversed: (3.14a) implies that $P_{t]} H_{[t}=H_{t}$, and 
(3.14b) implies that

$$
\left(Q X_{s} m,\left(1-P_{t}\right) X_{u} m^{\prime}\right)=\left(Q_{s} m, K(s, u) m^{\prime}\right)-\left(Q_{s} m, K(s, t) K(t, u) m^{\prime}\right),
$$

which vanishes by (3.14a), for all $m, m^{\prime}$ in $M$ and $s \leqq t \leqq u$ in $\mathbb{R}$; so that $Q H_{t]}$ is orthogonal to $D_{t}^{+}, Q$ maps $H_{t]}$ into itself, and commutes with $P_{t]}$, being skewadjoint. Then $A_{Q}\left(P_{t]}\right)$ exists and $A\left(P_{t]}\right) A\left(H_{[t}\right)=A\left(H_{t}\right)$.

In the special case of a stationary quasi-free Markov process, the semigroup $Z_{t}=j_{0}^{*} E_{0]} j_{t}$ is given by

$$
Z_{t}=A_{Q_{0}}\left(S_{t}\right), t \geqq 0,
$$

where $S_{t}=X_{0}^{*} X_{t}$, which is a semigroup by Equation (3.14a), and where $Q_{0}$ $=X_{0}^{*} Q X_{0}$, which is clearly a skew-adjoint contraction. Condition (3.7) holds by construction.

We prove a converse result.

Theorem 3.3.2. Let $\left\{Z_{t}=A_{Q_{0}}\left(S_{t}\right): t \geqq 0\right\}$ be a semigroup of quasi-free completely positive maps of $A(M)$. Then there is a stationary quasi-free Markov process over $A(M)$ such that $\left\{Z_{t}: t \geqq 0\right\}$ is the semigroup associated to $i t$, and the process is unique up to equivalence within the class of quasi-free Markov processes.

Proof. Define a covariance function by

$$
K(s, t)=\left\{\begin{array}{l}
S_{t-s}, t \geqq s, \\
S_{s-t}^{*}, t \leqq s,
\end{array}\right.
$$

and

$$
K^{Q}(s, t)=\left\{\begin{array}{l}
-Q_{0} S_{t-s}, t \geqq s, \\
-S_{s-t}^{*} Q_{0}, t \leqq s .
\end{array}\right.
$$

Clearly $K(s, t)$ and $K^{Q}(s, t)$ satisfy conditions (3.11a), (3.14a), (3.14b). We prove that $(3.11 \mathrm{~b})$ holds. We have

$$
\begin{aligned}
\sum_{i, j} \bar{c}_{i} c_{j}\left\{\left(m_{i}, K\left(t_{i}, t_{j}\right) m_{j}\right)+i\left(m_{i}, K^{Q}\left(t_{i}, t_{j}\right) m_{j}\right)\right\} \\
=\sum_{\left\{i, j: t_{i} \leqq t_{j}\right\}} \bar{c}_{i} c_{j} \omega_{Q}\left(B\left(m_{i}\right) Z_{t_{j}-t_{i}}\left(B\left(m_{j}\right)\right)\right) \\
\quad+\sum_{\left\{i, j: t_{i}>t_{j}\right\}} \bar{c}_{i} c_{j} \omega_{Q}\left(Z_{t_{i}-t_{j}}\left(B\left(m_{i}\right)\right) B\left(m_{j}\right)\right) .
\end{aligned}
$$

From the proof of Lemma 3.1, we know that $\left\{Z_{t}: t \geqq 0\right\}$ is a semigroup of contractions on $\operatorname{lin}_{C}\{B(m): m \in M\}$ with respect to the semi-norm $\|x\|_{Q}^{2}=\omega_{Q}\left(x^{*} x\right)$. 
A semigroup of contractions on a Hilbert space determines a positive definite function [47] hence the expression (3.16) is non-negative. It follows that the covariance function defined by Equations (3.15a-d) determines (up to equivalence) a quasi-free Markov process, which is also stationary by the proof of Theorem 3.2.2. The semigroup $\left\{Z_{t}^{\prime}: t \geqq 0\right\}$ associated to the process satisfies $Z_{t}^{\prime} B(m)=B\left(S_{t} m\right)$ for all $m$ in $M$ and $\omega_{Q^{\circ}} Z_{t}^{\prime}=\omega_{Q}$ for all $t \geqq 0$; and it is not difficult to see that it is indeed $Z_{t}^{\prime}=A_{Q_{0}}\left(S_{t}\right)=Z_{t}$. Conversely, any quasi-free Markov process such that the associated semigroup is $A_{Q_{0}}\left(S_{t}\right)$ has $(3.15 \mathrm{a}-\mathrm{d})$ as its covariance function, which proves uniqueness up to equivalence.

3.4. We characterize the class of those quasi-free Markov processes which satisfy a quantum Langevin equation.

Theorem 3.4.1. Let $\left(\mathscr{A},\left\{j_{t}\right\}, \omega_{Q}\right)$ be a stationary quasi-free Markov process, with associated semigroup $\left\{A_{Q_{0}}\left(S_{t}\right): t \geqq 0\right\}$; let $\left\{S_{t}\right\}$ be strongly continuous with infinitesimal generator $G$. Then the following are equivalent:

(i) $\lim _{t \rightarrow \infty}\left\|\mathscr{A}_{Q_{0}}\left(S_{t}\right)(b)-\omega_{Q_{0}}(b) 1\right\|=0$ for all $b$ in $A(M)$;

(ii) there is a family $\left\{\xi_{t}^{G}: t \in \boldsymbol{R}\right\}$ of linear operators from $\mathscr{D}(G)$ into $H$ such that

$$
\left(\xi_{t}^{G} m, \xi_{t^{\prime}}^{G} m^{\prime}\right)=\left(t \wedge t^{\prime}\right)\left(-\left(G m, m^{\prime}\right)-\left(m, G m^{\prime}\right)\right)
$$

for all $t, t^{\prime}$ in $\boldsymbol{R}, m, m^{\prime}$ in $\mathscr{D}(G)$,

$$
H=\vee\left\{\xi_{t}^{G} m: t \in \boldsymbol{R}, m \in \mathscr{D}(G)\right\}
$$

and $\left\{j_{t}\right\}$ satisfies the following Langevin equation:

$$
j_{t}(B(m))-j_{s}(B(m))=\int_{s}^{t} j_{t}(B(G m)) d u+B\left(\left(\xi_{t}^{G}-\check{\zeta}_{s}^{G}\right) m\right)
$$

for all $s \leqq t$ in $\boldsymbol{R}, m$ in $\mathscr{D}(G)$.

Proof. From the explicit form $(3.8 \mathrm{a}, \mathrm{b})$ of $A_{Q_{0}}\left(S_{t}\right)$, we see that (i) holds if and only if $\lim _{t \rightarrow \infty}\left\|S_{t} m\right\|=0$ for all $m$ in $M$. By [48] Theorem 4.2, [13] Theorem 3.15 , this is equivalent to

$$
X_{t} m-X_{s} m=\int_{s}^{t} X_{u} G m d u+\left(\xi_{t}^{G}-\xi_{s}^{G}\right) m
$$

for all $s \leqq \imath$ in $\boldsymbol{R}, m$ in $\mathscr{D}(G)$, where $\left\{\xi_{t}^{G}: t \in \boldsymbol{R}\right\}$ is a family as described in (ii); the conclusion of the theorem follows since $B: H \rightarrow A(H)$ is linear and isometric.

Notice that $\left\{A_{Q_{0}}\left(S_{t}\right): t \geqq 0\right\}$ is strongly continuous; if we denote its infinitesimal generator by $L$ we have $B(m) \in \mathscr{D}(L)$ if and only if $m \in \mathscr{D}(G)$ and 
$L B(m)=B(G m)$, so that Equation (3.17) can be re-written as

$$
j_{t}(B(m))-j_{s}(B(m))=\int_{s}^{t} j_{u} L(B(m)) d u+B\left(\left(\xi_{t}^{G}-\xi_{s}^{G}\right) m\right) .
$$

We can produce non-trivial examples of stationary Markov processes which are not quasi-free by applying to a stationary quasi-free Markov process the perturbation theory which we have developed in Theorems 2.3 and 2.4.4. Then we have the following

Theorem 3.4.2. If $\left\{j_{t}\right\}$ satisfies the Langevin equation (3.17) and the operator $v=v^{*}$ defining the perturbation is an even element of $A(M)$, then the perturbed process $\left\{\tilde{J}_{t}\right\}$ satisfies the following Langevin equation:

$$
\begin{aligned}
\tilde{J}_{t}(B(m))-\tilde{J}_{0}(B(m)) & =\int_{0}^{t} \tilde{J}_{u}(B(G m)+i[v, B(m)]) d u+B\left(\left(\xi_{t}^{G}-\xi_{0}^{G}\right) m\right) \\
& =\int_{0}^{t} \tilde{J}_{u} \tilde{L}(B(m)) d u+B\left(\left(\xi_{t}^{G}-\xi_{0}^{G}\right) m\right)
\end{aligned}
$$

for all $m$ in $\mathscr{D}(G)$ and $t \geqq 0, \tilde{L}$ being the infinitesimal generator of the perturbed semigroup $\left\{\tilde{Z}_{t}: t \geqq 0\right\}$.

Proof. We use the notation of Lemma 2.4.1. By standard perturbation theory, we have

$$
\begin{aligned}
\tilde{J}_{t}(B(m)) & =\tilde{u}_{t} j_{0}(B(m))=u_{t} j_{0}(B(m))+\int_{0}^{t} \tilde{u}_{s}\left(\left[i j_{0}(v), u_{t-s} j_{0}(B(m))\right]\right) d s \\
& =j_{t}(B(m))+\int_{0}^{t} \tilde{u}_{s}\left(\left[i j_{0}(v), j_{t-s}(B(m))\right]\right) d s .
\end{aligned}
$$

We use the Langevin equation (3.17) to compute $j_{t}(B(m)), j_{t-s}(B(m))$. Since $j_{0}(v)$ is an even element of $A\left(H_{0}\right) \subseteq A\left(H_{0]}\right)$ and $\left(\xi_{t}^{G}-\xi_{0}^{G}\right) m$ is in the orthogonal complement of $H_{0]}[13,48], j_{0}(v)$ commutes with $B\left(\left(\xi_{t}^{G}-\xi_{0}^{G}\right) m\right)$. Remembering that $\tilde{j}_{0}=j_{0}$ we are left with

$$
\begin{aligned}
& \tilde{j}_{t}(B(m))-\tilde{\jmath}_{0}(B(m))=\int_{0}^{t} j_{u}(B(G m)) d u+B\left(\left(\xi_{t}^{G}-\zeta_{0}^{G}\right) m\right) \\
& \left.\quad+\int_{0}^{t} \tilde{u}_{s}\left(\left[i j_{0}(v), j_{0}(B(m))\right)\right]\right) d s+\int_{0}^{t} \int_{0}^{t-s} \tilde{u}_{s}\left(\left[i j_{0}(v), j_{u}(B(m))\right]\right) d u d s .
\end{aligned}
$$

The first and the last term on the right-hand side can be combined together as in equation (3.19), to give $\int_{0}^{t} \tilde{J}_{u}(B(G m)) d u$, and the third term on the right-hand side is $\int_{0}^{t}\left[i \tilde{\jmath}_{u}(v), \tilde{J}_{u}(B(m))\right] d u$. This proves (3.18). 


\section{Remarks}

To 3.2: Quasi-free processes are an analogue of classical Gaussian processes, in that they are completely determined by a Hilbert space structure. For analogous ideas for processes on the CCR algebra, see Lindblad [49], and [34].

To 3.3: Theorem 3.3.1 is an analogue of Doob's theorem [10]. Stationary quasi-free Markov processes have been constructed from a semigroup $\left\{Z_{t}: t \geqq 0\right\}$ by Schrader and Uhlenbrock [36], in the special case $Z_{t}=A_{0}\left(S_{t}\right)$. A similar construction has been used by Evans $[43,44]$ in his study of dilations of quasi-free semigroups satisfying $\left[Q_{0}, S_{t}\right]=0$.

It is conceivable that there are quasi-free processes which are Markov without conditional expectations; we do not investigate this problem here.

To 3.4: The Langevin equation (3.17) bears no reference to the state $\omega_{Q}$ in $\left(\mathscr{A},\left\{j_{t}\right\}, \omega_{Q}\right)$; given $\mathscr{B}$ and $\mathscr{A}$, it determines $\left\{j_{t}: t \in \boldsymbol{R}\right\}$ but different choices of $\omega_{Q}$ could make the resulting process Markovian or not, according to whether or not $Q$ commutes with the projections $\left\{P_{t]}: t \in \mathbb{R}\right\}$.

The automorphism group of the perturbed process described in Theorem 4.2 is quasi-free if and only if the map $B(m) \mapsto i[v, B(m)]$ is quasi-free.

\section{§4. Examples}

We sketch how model systems of the Hepp and Lieb type $[11,12]$ can be regarded as perturbations of quasi-free processes.

4.1. The elementary building block of this class of models is a fermion coupled to two infinite fermion reservoirs in the Fock vacuum state $\left(\Omega_{0}, \Omega_{0}\right)$ with a total Hamiltonian [12]

$$
\begin{aligned}
\hat{H}^{0}=\varepsilon a^{*} a & +\int_{\boldsymbol{R}}\left[B(\omega)^{*} B(\omega)+C(\omega)^{*} C(\omega)\right] \omega d \omega \\
& +\int_{\boldsymbol{R}}\left[g^{B} B(\omega)^{*} a+g^{c} C(\omega)^{*} a+h . c .\right] d \omega,
\end{aligned}
$$

where $a^{*}, a$ are the creation and annihilation operators of the fermion, and $B(\omega)^{\#}, C(\omega)^{\#}$ are the creation and annihilation improper operators of the fermion reservoirs. The above Hamiltonian is formal, but the evolution gen- 
erated by it can be defined rigorously as a limit of evolutions generated by regularized Hamiltonians. The reduced dynamics of the fermion is given by a dynamical semigroup $\left\{Z_{t}: t \geqq 0\right\}$ satisfying [12]

$$
Z_{t}(a)=e^{-i \varepsilon t-\gamma t} a, Z_{t}\left(a^{*} a\right)=e^{-2 \gamma t} a^{*} a+\left(1-e^{-2 \gamma t}\right) \eta 1
$$

where

$$
\gamma=\pi\left\{\left|g^{B}\right|^{2}+\left|g^{C}\right|^{2}\right\}>0, \eta=\pi \gamma^{-1}\left|g^{C}\right|^{2} \in[0,1] .
$$

Via a Bogoliubov transformation

$$
A(\omega)=\left(\left|g^{B}\right|^{2}+\left|g^{C}\right|^{2}\right)^{-1 / 2}\left[g^{B} B(\omega)-g^{C} C(\omega)^{*}\right], \omega \in \mathbb{R},
$$

the coupling between system and reservoir becomes

$$
(\gamma / \pi)^{1 / 2} \int_{\boldsymbol{R}}\left[A(\omega)^{*} a+a^{*} A(\omega)\right] d \omega
$$

and the algebra generated by $\left\{a^{\sharp}, A(\omega)^{\sharp}: \omega \in \mathbb{R}\right\}$ is globally invariant under the evolution generated by the Hamiltonian (4.1). This algebra can be regarded as the Clifford algebra over the real Hilbert space

$$
H=\left[\mathbb{R} \oplus L_{\mathbb{R}}^{2}(\mathbb{R}) \oplus J\left(\mathbb{R} \oplus L_{\mathbb{R}}^{2}(\mathbb{R})\right)\right],
$$

(that is, $\mathbb{C} \oplus L_{\mathbb{C}}^{2}(\mathbb{R})$ regarded as a real Hilbert space).

The algebra generated by $a, a^{*}$ is the Clifford algebra over $H_{0}=\mathbb{R} \oplus J \mathbb{R}$ which is generated by two independent normalized vectors $h_{0}, J h_{0}$; then we have

$$
B\left(h_{0}\right)=a^{*}+a, B\left(J h_{0}\right)=i a^{*}-i a .
$$

Let $Q=J(1-2 \eta)$; then $Q$ commutes with the projection $P_{0}$ of $H$ onto $H_{0}$ and $Q_{0}=P_{0} Q P_{0}$ has the same form. The quasi-free state $\omega_{Q}$ on $A(H)$ is $\omega_{Q_{0}} \otimes$ $\left(\Omega_{0}, \cdot \Omega_{0}\right) \uparrow \vee\left\{A(\omega)^{\sharp}: \omega \in \mathbb{R}\right\}$. We have

$$
Z_{t}=A_{Q_{0}}\left(S_{t}\right), t \geqq 0,
$$

where $\left\{S_{t}=\exp (J \varepsilon t-\gamma t): t \geqq 0\right\}$ is a semigroup of contractions on $H_{0}$ commuting with $Q_{0}$.

The evolution generated by the Hamiltonian (4.1) is of the form $A\left(T_{t}\right)$, where $\left\{T_{t}: t \in \mathbb{R}\right\}$ acting on $H$ is a minimal unitary dilation of $\left\{S_{t}: t \geqq 0\right\}$ which commutes with $J$, hence with $Q$. Also the projections $P_{t]}$ of $H$ onto $H_{t]}=$ $\vee\left\{T_{s} H_{0}: s \leqq t\right\}$ commute with $Q$ for all $t$ in $\mathbb{R}_{R}$. Hence the quasi-free state $\omega_{Q}$ on $A(H)$ is invariant under $A\left(T_{t}\right)$ and compatible with conditional expectations $A_{Q}\left(P_{t]}\right)$, and $P_{t]} H_{[t}=H_{t}$ for each $t$ since $\left\{S_{t}=P_{0} T_{t} P_{0}: t \geqq 0\right\}$ is a semigroup. 
By Theorems 3.2.2. and 3.3.1, we conclude that $\left(A(H),\left\{A\left(T_{t} P_{0}\right)\right\}, \omega_{Q}\right)$ is a stationary quasi-free Markov process over $A\left(H_{0}\right)$; moreover, it is the unique (up to equivalence) reconstruction of a quasi-free Markov process from the semigroup (4.2), by Theorem 3.3.2. It satisfies the Langevin equation (3.17) if and only if $\gamma$ is strictly positive.

4.2. There is no additional difficulty in considering a finite or infinite collection of non-interacting elementary fermion systems as above: in any case we have a stationary quasi-free Markov process, satisfying the Langevin equation (3.17) if and only if all the damping constants $\gamma_{k}$ of the elementary fermion systems are strictly positive.

Then an interaction among the fermions can be introduced, in finite volume, to begin with. Let $v$ be an even self-adjoint polynomial in the creation and annihilation operators $a_{k}^{*}, a_{k}$, or equivalently in the $B\left(h_{0}^{(k)}\right), B\left(J h_{0}^{(k)}\right), k$ $=1, \ldots, N$ being the index which labels the elementary fermion systems. By Theorem 2.4.4, there is a perturbed stationary Markov process whose associated semigroups $\left\{\tilde{Z}_{t}: t \geqq 0\right\}$ has the form

$$
\tilde{Z}_{t}=\exp \left[t\left(\sum_{k=1}^{N} L_{k}+i[v, \cdot]\right)\right],
$$

$L_{k}$ being the infinitesimal generator of the semigroup $\left\{Z_{t}^{(k)} \geqq 0\right\}$ for the $k$-th fermion, given by

$$
L_{k}\left(a_{k}\right)=\left(-i \varepsilon_{k}-\gamma_{k}\right) a_{k}, \quad L_{k}\left(a_{k}^{*} a_{k}\right)=-2 \gamma_{k}\left(a_{k}^{*} a_{k}-\eta_{k} 1\right) .
$$

By Theorem 3.4.2, the perturbed process satisfies the Langevin equation (3.18) if and only if all $\gamma_{k}$ are strictly positive.

4.3. Also the thermodynamic limit $N \rightarrow \infty$ of interacting open fermion systems can be studied. This is somewhat outside the main scope of this paper, so we confine ourselves to a few remarks. If the interaction is of finite range and translationally invariant, the techniques of Robinson [50], Streater and Wilde [51] can be used to prove that there is a limit evolution for the infinite system; it can also be shown that there are a limit stationary state and a limit stationary Markov process. If the interaction is of mean field type, the works of Hepp and Lieb $[11,12]$ give a description of the limit evolution of intensive observables in "classical" states. Then one could employ the technique of Bogoliubov $\mathrm{Jr}$. [52, 53] to obtain the thermodynamic limit of multi-time correlation functions in these states. They define a limiting process; however we expect this limiting 
process to be neither stationary nor Markov in general, but rather to exhibit the structure with time-dependent Hamiltonians described in the notes to Section 2.4 (cf. van Hemmen [54] Appendix).

The general problem of limits of non-commutative stochastic processes (thermodynamic limit, and scaling limits which might make a process Markovian) is of obvious interest. Some rigorous results and open problems for scaling limits of classical processes are described by Spohn in [55]; we cannot but expect that the analogous non-commutative problems are at least as hard as those.

\section{Appendix}

Example A.1 (Lindblad [33], rephrased). Let $\mathscr{B}$ be the algebra of all complex $2 \times 2$ matrices. For each $x$ in $\mathbb{R}$, let $\left\{\alpha_{t}^{x}: t \in \boldsymbol{R}\right\}$ be the group of *-automorphisms of $\mathscr{B}$ given by

$$
\alpha_{t}^{x}(b)=e^{i x t \sigma_{3}} b e^{-i x t \sigma_{3}}, b \in \mathscr{B}, t \in \boldsymbol{R} .
$$

Define a stochastic process over $\mathscr{B}$, indexed by $\mathbb{R}$, by giving its correlation kernels as

$$
w_{\mathbf{t}}(\mathbf{a} ; \mathbf{b})=\frac{\gamma}{\pi} \int_{-\infty}^{\infty} \frac{d x}{\gamma^{2}+\left(x-x_{0}\right)^{2}} \frac{1}{2} \operatorname{trace}\left[\alpha_{t_{1}}^{x}\left(a_{1}\right)^{*} \cdots \alpha_{t_{n}}^{x}\left(a_{n}\right)^{*} \alpha_{t_{n}}^{x}\left(b_{n}\right) \cdots \alpha_{t_{1}}^{x}\left(b_{1}\right)\right]
$$

where $x_{0} \in \boldsymbol{R}, \gamma>0$, for all $\mathbf{t}=\left(t_{1}, \ldots, t_{n}\right)$ in $\mathbf{T}, \mathbf{a}=\left(a_{1}, \ldots, a_{n}\right), \mathbf{b}=\left(b_{1}, \ldots, b_{n}\right)$ in $\mathscr{B}_{\mathbf{i}}$. The explicit form of $\left(\mathscr{A},\left\{j_{t}\right\}, \omega\right)$ is not needed; we only notice that $\mathscr{A}$ can be chosen to be a $W^{*}$-algebra, the $j_{t}$ are normal maps of $\mathscr{B}$ into $\mathscr{A}, \omega$ is a faithful normal trace on $\mathscr{A}$, and the process is stationary.

For all $a_{1}, a_{2}, b_{1}, b_{2}$ in $\mathscr{B}, t_{1} \leqq t_{2}$ in $\mathbb{R}$, we have

$$
w_{t_{1}, t_{2}}\left(a_{1}, a_{2} ; b_{1}, b_{2}\right)=\omega_{0}\left(a_{1}^{*} Z_{t_{2}-t_{1}}\left(a_{2}^{*} b_{2}\right) b_{1}\right),
$$

where $\omega_{0}(\cdot)=2^{-1} \operatorname{trace}(\cdot)$, and $\left\{Z_{t}: t \geqq 0\right\}$ is the semigroup with infinitesimal generator $L$ given by

$$
L(b)=-\frac{\gamma}{2}\left[\sigma_{3},\left[\sigma_{3}, b\right]\right]+i x_{0}\left[\sigma_{3}, b\right], b \in \mathscr{B},
$$

satisfying $\lim _{t \rightarrow \infty}\left\|Z_{t}\left(\sigma_{1}\right)\right\|=0$. Since the modular automorphism group associated to $\omega$ is trivial, the canonical maps $E_{s, t}$ are conditional expectations; hence, if the process were Markov, the regression relation would hold, and $w_{0, t, 2 t}\left(a_{0}, a_{1}, 1\right.$; $\left.b_{0}, b_{1}, \sigma_{1}\right)$ would vanish in the limit as $t \rightarrow \infty$ for all $a_{0}, a_{1}, b_{0}, b_{1}$ in $\mathscr{B}$. However, it is clear that 
$w_{0, t, 2 t}\left(a_{0}, \sigma_{1}, 1 ; b_{0}, \sigma_{1}, \sigma_{1}\right)=\omega_{0}\left(a_{0}^{*} \sigma_{1} b_{0}\right) \quad$ for all $a_{0}, b_{0}$ in $\mathscr{B}, t \geqq 0$ since $\sigma_{1} \exp \left(i x t \sigma_{3}\right) \sigma_{1}=\exp \left(-i x t \sigma_{3}\right)$ and $\sigma_{1}=\left(\sigma_{1}\right)^{3}$. This contradicts the regression relation, hence the process is not Markov.

Example A.2. Let $\mathscr{B}=\mathscr{C}(\{-1,1\})$, the algebra of functions on the twopoint set $\{-1,1\}$, spanned by the identity 1 and the function $f$, such that $f( \pm 1)$ $= \pm 1$. Let $\omega_{0}$ be the state on $\mathscr{B}$ given by $\omega_{0}(f)=2^{-1}(f(1)+f(-1))$, and let $\left\{Z_{t}: t \geqq 0\right\}$ be the semigroup of maps determined by

$$
Z_{t}(1)=1, Z_{t}(f)=e^{-\gamma t} f, \gamma>0, \quad \text { for all } t \geqq 0 .
$$

Then $Z_{t}$ preserves positivity and the identity, and $\omega_{0} \circ Z_{t}=\omega_{0}$. By the Kolmogorov-Daniell construction, there is a classical stationary Markov process over $\mathscr{B}$ such that the regression relation (2.9) holds. However, $\mathscr{B}$ may also be identified with the Clifford algebra $A(\mathbb{R})$, spanned by the identity 1 and a selfadjoint unitary $B(e)$ ( $e$ being a unit vector in $\mathbb{R}$ ) which corresponds to $f$. If this identification is made, $\omega_{0}$ is the quasi-free state corresponding to $Q=0$, and $\left\{Z_{t}\right\}$ is the quasi-free semigroup given by $Z_{t}=A_{0}\left(e^{-\gamma t}\right)$. Let $\left\{X_{t}: \mathbb{R} \rightarrow H ; t \in \mathbb{R}\right\}$ be a minimal Kolmogorov decomposition of the positive definite kernel $s, t$ $\mapsto \exp (-\gamma|t-s|)$, for instance, $H=L^{2}(\mathbb{R}), X_{t} r=(2 \gamma)^{1 / 2} \exp (-\gamma|t-s|) \chi_{(-\infty, t]}(s) r$, $r \in \mathbb{R}$ [13]. Let $j_{t}(B(r))=B\left(X_{t} r\right) \in A(H)$, and let $\omega_{0}$ be the quasi-free state on $A(H)$ determined by $Q=0$ on $H$; then $\left(A(H),\left\{j_{t}\right\}, \omega_{0}\right)$ is a stationary quasifree Markov process with conditional expectations, by Theorem 3.3.1; hence the regression relation (2.9) holds. Obviously, $A(H)$ cannot be isomorphic to an abelian algebra.

\section{References}

[1] Doob, J. L., Stochastic processes, Wiley, New York, 1953.

[2] Meyer, P. A., Probability and Potential, Blaisdell, Waltham, Mass., 1966.

[3] Accardi, L., Non-relativistic quantum mechanics as a non-commutative Markov process, Advances in Math., 20 (1976), 329-366.

[4] Haag, R. and Kastler, D., An algebraic approach to quantum field theory, J. Math. Phys., 5 (1964), 848-861.

[5] Glauber, R. J., Optical coherence and photon statistics, Quantum Optics and Electronics, Les Houches 1964, edited by C. De Witt et al., Gordon and Breach, New York, 1965, 63-185.

[6] Davies, E. B., Quantum theory of open systems, Academic Press, London, 1976.

[7] Kolmogorov, A. N., Grundbegriffe der Wahrscheinlichkeitsrechnung, Springer, Berlin, 1933.

[8] Accardi, L., On the quantum Feynman-Kac formula, Rendiconti del Seminario 
Matematico e Fisico di Milano, 48 (1978), 135-180 (lecture given in 1978, paper actually printed in 1980).

[9] Accardi, L., A quantum formulation of the Feynman-Kac formula, Proceedings of the Colloquium "Random fields: rigorous results in siatistical mechanics and quantum field theory", Esztergom, 1979, to appear.

[10] Doob, J. L., The elementary Markov processes, Ann. Math. Stat., 15 (1944), 229-282.

[11] Hepp, K. and Lieb, E. H., Phase transitions in reservoir-driven open systems, with applications to lasers and superconductors, Helv. Phys. Acta, 46 (1973), 575-603.

[12] Hepp, K. and Lieb, E. H., The laser: a reversible quantum dynamical system with irreversible classical macroscopic motion, Lecture notes in Physics, 38, Springer, BerlinHeidelberg-New York, 1975, 179-207.

[13] Evans, D. E. and Lewis, J. T., Dilations of irreversible evolutions in algebraic quantum theory, Commun. Dublin Institute for Advanced Studies, Ser. A, 24 (1977).

[14] Segal, I. E., Equivalence of measure spaces, Amer. J. Math., 73 (1951), 274-313.

[15] Gelfand, I. M. and Vilenkin, N. Ya., Generalized Functions, vol. 4, Applications of Harmonic Analysis, Academic Press, New' York, 1964.

[16] von Neumann, J., Mathematical Foundations of Quantum Mechanics, Princeton University Press, Princeton, N. J., 1955.

[17] Wightman, A. S., Quantum field theory in terms of vacuum expectations values, Phys. Rev., 101 (1956), 860-866.

[18] Dubin, D. A. and Sewell, G. L., Time translations in the algebraic formulation of statistical mechanics, J. Math. Phys., $\mathbb{1 1}$ (1970), 2990-2998.

[19] Winnink, M., Some general properties of thermodynamic states in an algebraic approach, Haifa Summer School, Statistical Mechanics and Field Theory, ed. R. N. Sen and C. Weil, Halsted Press, New York, 1971.

[20] Accardi, L., Non-commutative Markov chains associated to a pre-assigned evolution, an application to the quantum theory of measurement, Advances in Math., 29 (1978), 226-243.

[21] Accardi, L. and Cecchini, C., Conditional expectations on $W^{*}$-algebras and a theorem of Takesaki, preprint 1980.

[22] Takesaki, M., Conditional expectations in von Neumann algebras, J. Funct. Anal., 9 (1972), 306-321.

[23] Accardi, L., On the non-commutative Markov property, Funct. Anal. Appl., 9 (1975), 1-8 (in Russian).

[24] Accardi, L., Non-commutative Markov chains, Proceedings Summer School in Mathematical Physics, Camerino, 1974.

[25] Lax, M., Quantum noise XI, Multi-time correspondence between quantum and classical stochastic processes, Phys. Rev., 172 (1968), 350-361.

[26] Haake, F., Density operator and multi-time correlation functions for open systems, Phys. Rev., A3 (1971), 1723-1734.

[27] Lugiato, L., Generalized regression theorem for open systems, Physica, 85 A (1976), $18-27$.

[28] Robinson, D. W., Return to equilibrium, Commun. math. Phys., 31 (1973), 171-189.

[29] Nelson, E., Construction of quantum fields from Markov fields, J. Funct. Anal., 12 (1973), 97-112; The free Markov field, ibidem, $\mathbb{1 2}$ (1973), 211-277.

[30] Guerra, F., Rosen, L. and Simon, B., The $P(\varphi)_{2}$ Euclidean quantum field theory as classical statistical mechanics, Ann. Math., 101 (1975), 111-259.

[31] Lindblad, G., Non-Markovian quantum stochastic processes and their entropy, Commun. math. Phys., 65 (1979), 281-294. 
[32] Lévy, P., Exemples de processus pseudo-Markoviens, Compt. Rend. Acad. Sci. Paris, 228 (1949), 2004-2006.

[33] Lindblad, G., Response of Markovian and non-Markovian quantum stochastic systems to time-dependent forces, preprint, Stockholm, 1980.

[34] Frigerio, A. and Lewis, J. T., Non-commutative Gaussian processes, preprint DIAS-STP-80-02, Dublin, 1980.

[35] Osterwalder, K. and Schrader, R., Euclidean Fermi fields and a Feynman-Kac formula for Boson-Fermion models, Helv. Phys. Acta, 46 (1973), 277-302.

[36] Schrader, R. and Uhlenbrock, D. A., Markov structures on Clifford algebras, $J$. Funct. Anal., 18 (1975), 369-413.

[37] Hudson, R. L. and Ion, P. D. F., The Feynman-Kac formula for a canonical quantummechanical Wiener process, Proceedings of the Colloquium "Randon Fields; rigorous results in statistical mechanics and quantum field theory", Esztergom, 1979, to appear.

[38] Hudson, R. L., Ion, P. D. F. and Parthasarathy, K. R., Time-orthogonal unitary dilations and noncommutative Feynman-Kac formulae, Commun. math. Phys., 83 (1982), 261-280.

[39] Klein, L. and Landau, L. J., Singular perturbations of positivity preserving semigroups via path space techniques, J. Funct. Anal., 20 (1975), 44-82.

[40] Shale, D. and Stinespring, W. F., States on the Clifford algebra, Ann. Math., 80 (1964), 365-381.

[41] Balslev, E., Manuceau, J. and Verbeure, A., Representations of anti-commutation relations and Bogoliubov transformations, Commun. math. Phys., 8 (1968), 315-326.

[42] Manuceau, J., Rocca, F. and Testard, D., On the product form of quasi-free states, Commun. math. Phys., 12 (1969), 43-57.

[43] Evans, D. E., Completely positive quasi-free maps on the CAR algebra, Commun. math. Phys., 70 (1979), 53-68.

[44] __ Completely positive quasi-free maps on the fermion algebra, Mathematical problems in the quantum theory of irreversible processes, ed. L. Accardi, V. Gorini, G. Parravicini, Proc. Arco Felice (Naples) Conference, 1978 (1979).

[45] Fannes, M. and Rocca, F., A class of dissipative evolutions with applications in thermodynamics of fermion systems, J. Math. Phys., 21 (1980), 221-226.

[46] Rocca, F., Sirugue, M. and Testard, D., On a class of equilibrium states under the Kubo-Martin-Schwinger boundary condition, I, Fermions, Commun. math. Phys., 13 (1969), 317-334.

[47] Sz.-Nagy, B. and Foias, C., Harmonic analysis of operators on Hilbert space, North Holland, Amsterdam, 1970 (page 29).

[48] Lewis, J. T. and Thomas, L. C., A characterization of regular solutions of linear stochastic differential equation, Z.f. Wahrscheinlichkeitstheorie, 30 (1974), 45-55.

[49] Lindblad, G., Gaussian quantum stochastic processes on the CCR algebra, J. Math. Phys., 20 (1979), 2081-2087.

[50] Robinson, D. W., Statistical mechanics of quantum spin systems II, Commun. math. Phys., 7 (1968), 337-340.

[51] Streater, R. F. and Wilde, I. F., The time evolution of quantized fields with bounded quasi-local interaction density, Commun. math. Phys., 17 (1970), 21-32.

[52] Bogoliubov, N. N. (Jr.), A method for studying model Hamiltonians, Pergamon Press, Oxford, 1972.

[53] —_, Generalized theorems in model system theory, Commun. Joint Institute for Nuclear Research D 17-10148, Dubna, 1977.

[54] Van Hemmen, L., Linear fermion systems, molecular field models and the KMS 
condition, preprint IHES/P/77/174, 1977.

[55] Spohn, H., Kinetic equations from Hamiltonian dynamics, the Markovian limits, Reviews of Modern Physics, 52 (1980), 569-615. 
